\title{
Bifurcation and Global Dynamics of a Leslie-Gower Type Competitive System of Rational Difference Equations with Quadratic Terms
}

\author{
V. Hadžiabdić, ${ }^{1}$ M. R. S. Kulenović, ${ }^{2}$ and E. Pilav ${ }^{3}$ \\ ${ }^{1}$ Division of Mathematics, Faculty of Mechanical Engineering, University of Sarajevo, Bosnia and Herzegovina \\ ${ }^{2}$ Department of Mathematics, University of Rhode Island, Kingston, RI 02881-0816, USA \\ ${ }^{3}$ Department of Mathematics, University of Sarajevo, 75000 Sarajevo, Bosnia and Herzegovina
}

Correspondence should be addressed to M. R. S. Kulenović; mkulenovic@mail.uri.edu

Received 1 April 2017; Accepted 4 June 2017; Published 2 August 2017

Academic Editor: Patricia J. Y. Wong

Copyright (C) 2017 V. Hadžiabdić et al. This is an open access article distributed under the Creative Commons Attribution License, which permits unrestricted use, distribution, and reproduction in any medium, provided the original work is properly cited.

We investigate global dynamics of the following systems of difference equations $x_{n+1}=x_{n} /\left(A_{1}+B_{1} x_{n}+C_{1} y_{n}\right), y_{n+1}=y_{n}^{2} /\left(A_{2}+\right.$ $\left.B_{2} x_{n}+C_{2} y_{n}^{2}\right), n=0,1, \ldots$, where the parameters $A_{1}, A_{2}, B_{1}, B_{2}, C_{1}$, and $C_{2}$ are positive numbers and the initial conditions $x_{0}$ and $y_{0}$ are arbitrary nonnegative numbers. This system is a version of the Leslie-Gower competition model for two species. We show that this system has rich dynamics which depends on the part of parametric space.

\section{Introduction}

In this paper we study the global dynamics of the following rational system of difference equations:

$$
\begin{aligned}
& x_{n+1}=\frac{x_{n}}{A_{1}+B_{1} x_{n}+C_{1} y_{n}}, \\
& y_{n+1}=\frac{y_{n}^{2}}{A_{2}+B_{2} x_{n}+C_{2} y_{n}^{2}},
\end{aligned}
$$

$$
n=0,1, \ldots,
$$

where the parameters $A_{1}, A_{2}, B_{1}, B_{2}, C_{1}$, and $C_{2}$ are positive numbers and initial conditions $x_{0}$ and $y_{0}$ are arbitrary nonnegative numbers.

System (1) is a competitive system, and our results are based on recent results about competitive systems in the plane; see [1]. System (1) can be used as a mathematical model for competition in population dynamics. System (1) is related to Leslie-Gower competition model

$$
\begin{aligned}
& x_{n+1}=\frac{x_{n}}{A_{1}+B_{1} x_{n}+C_{1} y_{n}}, \\
& y_{n+1}=\frac{y_{n}}{A_{2}+B_{2} x_{n}+C_{2} y_{n}}, \\
& n=0,1, \ldots,
\end{aligned}
$$

where the parameters $A_{1}, A_{2}, B_{1}, B_{2}, C_{1}$, and $C_{2}$ are positive numbers and initial conditions $x_{0}$ and $y_{0}$ are arbitrary nonnegative numbers, considered in [2]. System (2) globally exhibits three dynamic scenarios in five parametric regions which are competitive exclusion, competitive coexistence, and existence of an infinite number of equilibrium solutions; see [1-3]. System (2) does not exhibit the Allee effect, which is desirable from modeling point of view. The simplest variation of system (2) which exhibits the Allee effect is probably system

$$
\begin{aligned}
x_{n+1}=\frac{x_{n}^{2}}{A_{1}+B_{1} x_{n}^{2}+C_{1} y_{n}}, & \\
y_{n+1}=\frac{y_{n}^{2}}{A_{2}+B_{2} x_{n}+C_{2} y_{n}^{2}}, & \\
& n=0,1, \ldots,
\end{aligned}
$$

where the parameters $A_{1}, A_{2}, B_{1}, B_{2}, C_{1}$, and $C_{2}$ are positive numbers and initial conditions $x_{0}$ and $y_{0}$ are arbitrary nonnegative numbers, considered in [4]. System (3) has between 1 and 9 equilibrium points and exhibits nine dynamics scenarios part of each is the Allee effect. In the case of the dynamic 
scenario with nine equilibrium points system (3) exhibits both competitive exclusion and competitive coexistence as well as the Allee effect. Another system with quadratic terms is

$$
\begin{aligned}
& x_{n+1}=\frac{x_{n}^{2}}{B_{1} x_{n}^{2}+C_{1} y_{n}^{2}}, \\
& y_{n+1}=\frac{y_{n}^{2}}{A_{2}+B_{2} x_{n}^{2}+C_{2} y_{n}^{2}}, \\
& \qquad n=0,1, \ldots,
\end{aligned}
$$

where the parameters $A_{2}, B_{1}, B_{2}, C_{1}$, and $C_{2}$ are positive numbers and initial conditions $x_{0}$ and $y_{0}$ are arbitrary nonnegative numbers such that $x_{0}+y_{0}>0$, considered in [5]. System (4) exhibits seven scenarios part of each is singular Allee's effect, which means that the origin as the singular point of this system still has some basin of attraction. First systematic study for a system with quadratic terms was performed in [6] for system

$$
\begin{aligned}
x_{n+1}= & \frac{x_{n}}{A_{1}+y_{n}^{2}}, \\
y_{n+1}=\frac{y_{n}}{A_{2}+x_{n}^{2}}, & \\
n & \quad n=0,1, \ldots
\end{aligned}
$$

which exhibits nine dynamic scenarios and whose dynamics is very similar to the corresponding system without quadratic terms considered in [7].

In general, it seems that an introduction of quadratic terms in equations of the Leslie-Gower model (2) generates the Allee effect. We will test this hypothesis in this paper by introducing the quadratic terms only in the second equation. System (1) can be considered as the competitive version of the decoupled system

$$
\begin{aligned}
x_{n+1}= & \frac{x_{n}}{A_{1}+B_{1} x_{n}}, \\
y_{n+1}=\frac{y_{n}^{2}}{A_{2}+C_{2} y_{n}^{2}}, & \\
& n=0,1, \ldots,
\end{aligned}
$$

where the parameters $A_{1}, A_{2}, B_{1}$, and $C_{2}$ are positive numbers and initial conditions $x_{0}$ and $y_{0}$ are arbitrary nonnegative numbers, whose dynamics can be directly obtained from two separate equations. Unlike system (2) which has five regions of parameters with distinct local behavior system (1) has eighteen regions of parameters with distinct local behavior, which is caused by the geometry of the problem, that is, by the geometry of equilibrium curves. More precisely, the equilibrium curves of system (2) are lines while the equilibrium curves of system (1) are a line and a parabola. In the case when $A_{1}>1$, all equilibrium points are hyperbolic and all solutions are attracted to the three equilibrium points on the $y$-axis and we can describe this situation as competitive exclusion case. When $A_{1}=1$, the equilibrium point $E_{1}$ is nonhyperbolic and dynamics is analogous to the case when $A_{1}>1$. In both cases the Allee effect is present. When $A_{1}<1$, there exist 11 regions of parameters with different global dynamics. In nine of these regions the global dynamics is in competitive exclusion case, which means that all solutions converge to one of the equilibrium points on the axes and in only two situations we have competitive coexistence case, which means that the interior equilibrium points have substantial basin of attraction. In all 11 cases, the zero equilibrium has some basin of attraction which is a part of $y$-axis so we can say that in these cases system (1) exhibits weak Allee's effect. Figure 3 gives the bifurcation diagram showing the transition from different global dynamics situations when $A_{1}<1$, since the cases $A_{1} \geq 1$ are simple and do not need graphical interpretation.

The paper is organized as follows. Section 2 contains some necessary results on competitive systems in the plane. Section 3 provides some basic information about the number of equilibrium points. Section 4 contains local stability analysis of all equilibrium solutions. Section 5 contains some global results on injectivity of the map associated with system (1). Section 6 gives global dynamics of system (1) in all regions of the parameters.

\section{Preliminaries}

A first-order system of difference equations

$$
\begin{aligned}
& x_{n+1}=f\left(x_{n}, y_{n}\right), \\
& y_{n+1}=g\left(x_{n}, y_{n}\right), \\
& \quad n=0,1, \ldots,
\end{aligned}
$$

where $\mathcal{S} \subset \mathbb{R}^{2},(f, g): \mathcal{S} \rightarrow \mathcal{S}, f, g$ are continuous functions is competitive if $f(x, y)$ is nondecreasing in $x$ and nonincreasing in $y$, and $g(x, y)$ is nonincreasing in $x$ and nondecreasing in $y$. If both $f$ and $g$ are nondecreasing in $x$ and $y$, system (7) is cooperative. Competitive and cooperative maps are defined similarly. Strongly competitive systems of difference equations or strongly competitive maps are those for which the functions $f$ and $g$ are coordinate-wise strictly monotone.

Competitive and cooperative systems have been investigated by many authors; see [1-3, 7-16]. Special attention to discrete competitive and cooperative systems in the plane was given in $[1-3,16,17]$. One of the reasons for paying special attention to two-dimensional discrete competitive and cooperative systems is their applicability and the fact that many examples of mathematical models in biology and economy which involve competition or cooperation are models which involve two species. Another reason is that the theory of two-dimensional discrete competitive and cooperative systems is very well developed, unlike such theory for three-dimensional and higher systems. Part of the reason for this situation is de Mottoni-Schiaffino theorem given below, which provides relatively simple scenarios for possible behavior of many two-dimensional discrete competitive and cooperative systems. However, this does not mean that one 
can not encounter chaos in such systems as has been shown by Smith; see [16].

If $\mathbf{v}=(u, v) \in \mathbb{R}^{2}$, we denote with $Q_{\ell}(\mathbf{v}), \ell \in\{1,2,3,4\}$, the four quadrants in $\mathbb{R}^{2}$ relative to $\mathbf{v}$, that is, $\mathbb{Q}_{1}(\mathbf{v})=\{(x, y) \in$ $\left.\mathbb{R}^{2}: x \geq u, y \geq v\right\}, \mathbb{Q}_{2}(\mathbf{v})=\left\{(x, y) \in \mathbb{R}^{2}: x \leq\right.$ $u, y \geq v\}$, and so on. Define the South-East partial order $\preceq_{\text {se }}$ on $\mathbb{R}^{2}$ by $(x, y) \preceq_{\text {se }}(s, t)$ if and only if $x \leq s$ and $y \geq t$. Similarly, we define the North-East partial order $\preceq_{\mathrm{ne}}$ on $\mathbb{R}^{2}$ by $(x, y) \preceq_{\mathrm{ne}}(s, t)$ if and only if $x \leq s$ and $y \leq t$. For $\mathscr{A} \subset \mathbb{R}^{2}$ and $x \in \mathbb{R}^{2}$, define the distance from $x$ to $\mathscr{A}$ as $\operatorname{dist}(x, \mathscr{A}):=$ $\inf \{\|x-y\|: y \in \mathscr{A}\}$. By int $\mathscr{A}$ we denote the interior of a set $\mathscr{A}$.

It is easy to show that a map $F$ is competitive if it is nondecreasing with respect to the South-East partial order, that is, if the following holds:

$$
\begin{gathered}
\left(\begin{array}{c}
x^{1} \\
y^{1}
\end{array}\right) \preceq_{\mathrm{se}}\left(\begin{array}{c}
x^{2} \\
y^{2}
\end{array}\right) \Longrightarrow \\
F\left(\begin{array}{c}
x^{1} \\
y^{1}
\end{array}\right) \preceq_{\mathrm{se}} F\left(\begin{array}{c}
x^{2} \\
y^{2}
\end{array}\right) .
\end{gathered}
$$

For standard definitions of attracting fixed point, saddle point, stable manifold, and related notions see [10].

We now state three results for competitive maps in the plane. The following definition is from [16].

Definition 1. Let $\mathcal{S}$ be a nonempty subset of $\mathbb{R}^{2}$. A competitive map $T: \mathcal{S} \rightarrow \mathcal{S}$ is said to satisfy condition $(O+)$ if for every $x$, $y$ in $\mathcal{S}, T(x) \preceq_{\mathrm{ne}} T(y)$ implies $x \preceq_{\mathrm{ne}} y$, and $T$ is said to satisfy condition (O-) if for every $x, y$ in $\mathcal{S}, T(x) \preceq_{\mathrm{ne}} T(y)$ implies $y \preceq_{\mathrm{ne}} x$.

The following theorem was proved by de MottoniSchiaffino [17] for the Poincaré map of a periodic competitive Lotka-Volterra system of differential equations. Smith generalized the proof to competitive and cooperative maps $[13,14]$.

Theorem 2. Let $\mathcal{S}$ be a nonempty subset of $\mathbb{R}^{2}$. If $T$ is a competitive map for which $(\mathrm{O}+)$ holds then for all $x \in \mathcal{S}$, $\left\{T^{n}(x)\right\}$ is eventually componentwise monotone. If the orbit of $x$ has compact closure, then it converges to a fixed point of $T$. If instead (O-) holds, then for all $x \in \mathcal{S},\left\{T^{2 n}(x)\right\}$ is eventually componentwise monotone. If the orbit of $x$ has compact closure in $\mathcal{S}$, then its omega limit set is either a period-two orbit or a fixed point.

The following result is from [16], with the domain of the map specialized to be the Cartesian product of intervals of real numbers. It gives a sufficient condition for conditions $(\mathrm{O}+)$ and $(\mathrm{O}-)$.

Theorem 3. Let $\mathscr{R} \subset \mathbb{R}^{2}$ be the Cartesian product of two intervals in $\mathbb{R}$. Let $T: \mathscr{R} \rightarrow \mathscr{R}$ be a $C^{1}$ competitive map. If $T$ is injective and $\operatorname{det} J_{T}(x)>0$ for all $x \in \mathscr{R}$ then $T$ satisfies $(O+)$. If $T$ is injective and det $J_{T}(x)<0$ for all $x \in \mathscr{R}$ then $T$ satisfies $(\mathrm{O}-)$.
The following result is a direct consequence of the Trichotomy Theorem of Dancer and Hess (see [18]) and is helpful for determining the basins of attraction of the equilibrium points.

Corollary 4. If the nonnegative cone of $\preceq$ is a generalized quadrant in $\mathbb{R}^{n}$, and if $T$ has no fixed points in $\llbracket u_{1}, u_{2} \rrbracket$ other than $u_{1}$ and $u_{2}$, then the interior of $\llbracket u_{1}, u_{2} \rrbracket$ is either a subset of the basin of attraction of $u_{1}$ or a subset of the basin of attraction of $u_{2}$.

Next result is well known global attractivity result which holds in partially ordered Banach spaces as well; see [18].

Theorem 5. Let $T$ be a monotone map on a closed and bounded rectangular region $\mathscr{R} \subset \mathbb{R}^{2}$. Suppose that $T$ has a unique fixed point $\overline{\mathbf{e}}$ in $\mathscr{R}$. Then $\overline{\mathbf{e}}$ is a global attractor of $T$ on $\mathscr{R}$.

The following theorems were proved by Kulenović and Merino [1] for competitive systems in the plane, when one of the eigenvalues of the linearized system at an equilibrium (hyperbolic or nonhyperbolic) is by absolute value smaller than 1 while the other has an arbitrary value. These results are useful for determining basins of attraction of fixed points of competitive maps.

Theorem 6. Let $T$ be a competitive map on a rectangular region $\mathscr{R} \subset \mathbb{R}^{2}$. Let $\bar{x} \in \mathscr{R}$ be a fixed point of $T$ such that $\Delta:=\mathscr{R} \cap \operatorname{int}\left(\mathbb{Q}_{1}(\bar{x}) \cup \mathbb{Q}_{3}(\bar{x})\right)$ is nonempty (i.e., $\bar{x}$ is not the $N W$ or $S E$ vertex of $\mathscr{R})$, and $T$ is strongly competitive on $\Delta$. Suppose that the following statements are true:

(a) The map $T$ has a $C^{1}$ extension to a neighborhood of $\bar{x}$.

(b) The Jacobian $J_{T}(\bar{x})$ of T at $\bar{x}$ has real eigenvalues $\lambda, \mu$ such that $0<|\lambda|<\mu$, where $|\lambda|<1$, and the eigenspace $E^{\lambda}$ associated with $\lambda$ is not a coordinate axis.

Then there exists a curve $\mathscr{C} \subset \mathscr{R}$ through $\bar{x}$ that is invariant and a subset of the basin of attraction of $\bar{x}$, such that $\mathscr{C}$ is tangential to the eigenspace $E^{\lambda}$ at $\bar{x}$, and $\mathscr{C}$ is the graph of a strictly increasing continuous function of the first coordinate on an interval. Any endpoints of $\mathscr{C}$ in the interior of $\mathscr{R}$ are either fixed points or minimal period-two points. In the latter case, the set of endpoints of $\mathscr{C}$ is a minimal period-two orbit of $T$.

The situation where the endpoints of $\mathscr{C}$ are boundary points of $\mathscr{R}$ is of interest. The following result gives a sufficient condition for this case.

Theorem 7. For the curve $\mathscr{C}$ of Theorem 6 to have endpoints in $\partial \mathscr{R}$, it is sufficient that at least one of the following conditions is satisfied

(i) The map $T$ has no fixed points or periodic points of minimal period-two in $\Delta$.

(ii) The map $T$ has no fixed points in $\Delta$, det $J_{T}(\bar{x})>0$, and $T(x)=\bar{x}$ has no solutions $x \in \Delta$.

(iii) The map $T$ has no points of minimal period-two in $\Delta$, $\operatorname{det} J_{T}(\bar{x})<0$, and $T(x)=\bar{x}$ has no solutions $x \in \Delta$. 
The next result is useful for determining basins of attraction of fixed points of competitive maps.

Theorem 8. (A) Assume the hypotheses of Theorem 6, and let $\mathscr{C}$ be the curve whose existence is guaranteed by Theorem 6. If the endpoints of $\mathscr{C}$ belong to $\partial \mathscr{R}$, then $\mathscr{C}$ separates $\mathscr{R}$ into two connected components, namely,

$$
\begin{aligned}
& \mathscr{W}_{-}:=\left\{x \in \mathscr{R} \backslash \mathscr{C}: \exists y \in \mathscr{C} \text { with } x \preceq_{s e} y\right\}, \\
& \mathscr{W}_{+}:=\left\{x \in \mathscr{R} \backslash \mathscr{C}: \exists y \in \mathscr{C} \text { with } y \preceq_{s e} x\right\},
\end{aligned}
$$

such that the following statements are true:

(i) $\mathscr{W}_{-}$is invariant, and $\operatorname{dist}\left(T^{n}(x), \mathcal{Q}_{2}(\bar{x})\right) \rightarrow 0$ as $n \rightarrow$ $\infty$ for every $x \in \mathscr{W}_{-}$.

(ii) $\mathscr{W}_{+}$is invariant, and $\operatorname{dist}\left(T^{n}(x), Q_{4}(\bar{x})\right) \rightarrow 0$ as $n \rightarrow$ $\infty$ for every $x \in \mathscr{W}_{+}$.

(B) If, in addition to the hypotheses of part (A), $\bar{x}$ is an interior point of $\mathscr{R}$ and $T$ is $C^{2}$ and strongly competitive in a neighborhood of $\bar{x}$, then $T$ has no periodic points in the boundary of $Q_{1}(\bar{x}) \cup Q_{3}(\bar{x})$ except for $\bar{x}$, and the following statements are true:

(iii) For every $x \in \mathscr{W}_{-}$there exists $n_{0} \in \mathbb{N}$ such that $T^{n}(x) \in$ int $Q_{2}(\bar{x})$ for $n \geq n_{0}$.

(iv) For every $x \in \mathscr{W}_{+}$there exists $n_{0} \in \mathbb{N}$ such that $T^{n}(x) \in$ int $Q_{4}(\bar{x})$ for $n \geq n_{0}$.

If $T$ is a map on a set $\mathscr{R}$ and if $\bar{x}$ is a fixed point of $T$, the stable set $\mathscr{W}^{s}(\bar{x})$ of $\bar{x}$ is the set $\left\{x \in \mathscr{R}: T^{n}(x) \rightarrow \bar{x}\right\}$ and unstable set $\mathscr{W}^{u}(\bar{x})$ of $\bar{x}$ is the set

$$
\begin{aligned}
& \left\{x \in \mathscr{R}: \text { there exists }\left\{x_{n}\right\}_{n=-\infty}^{0} \subset \mathscr{R} \text { s.t. } T\left(x_{n}\right)\right. \\
& \left.=x_{n+1}, x_{0}=x, \lim _{n \rightarrow-\infty} x_{n}=\bar{x}\right\} .
\end{aligned}
$$

When $T$ is noninvertible, the set $\mathscr{W}^{s}(\bar{x})$ may not be connected and made up of infinitely many curves, or $\mathscr{W}^{u}(\bar{x})$ may not be a manifold. The following result gives a description of the stable and unstable sets of a saddle point of a competitive map. If the map is a diffeomorphism on $\mathscr{R}$, the sets $\mathscr{W}^{s}(\bar{x})$ and $\mathscr{W}^{u}(\bar{x})$ are the stable and unstable manifolds of $\bar{x}$.

Theorem 9. In addition to the hypotheses of part (B) of Theorem 8, suppose that $\mu>1$ and that the eigenspace $E^{\mu}$ associated with $\mu$ is not a coordinate axis. If the curve $\mathscr{C}$ of Theorem 6 has endpoints in $\partial \mathscr{R}$, then $\mathscr{C}$ is the stable set $\mathscr{W}^{s}(\bar{x})$ of $\bar{x}$, and the unstable set $\mathscr{W}^{u}(\bar{x})$ of $\bar{x}$ is a curve in $\mathscr{R}$ that is tangential to $E^{\mu}$ at $\bar{x}$ and such that it is the graph of a strictly decreasing function of the first coordinate on an interval. Any endpoints of $\mathscr{W}^{u}(\bar{x})$ in $\mathscr{R}$ are fixed points of $T$.

\section{Number of Equilibria}

In this section we give some basic facts which are used later. Let $T$ be the map associated with system (1) given by

$$
\begin{aligned}
T(x, y) & =(f(x, y), g(x, y)) \\
& =\left(\frac{x}{A_{1}+B_{1} x+C_{1} y}, \frac{y^{2}}{A_{2}+B_{2} x+C_{2} y^{2}}\right) .
\end{aligned}
$$

Let $\mathscr{R}=\mathbb{R}_{+}^{2}$. The equilibrium points $(\bar{x}, \bar{y})$ of system (1) satisfy equations

$$
\begin{aligned}
& \frac{\bar{x}}{A_{1}+B_{1} \bar{x}+C_{1} \bar{y}}=\bar{x}, \\
& \frac{\bar{y}^{2}}{A_{2}+B_{2} \bar{x}+C_{2} \bar{y}^{2}}=\bar{y} .
\end{aligned}
$$

For $\bar{x}=0$ we have

$$
\bar{y}=\bar{y}^{2}-A_{2} \bar{y}-C_{2} \bar{y}^{3}
$$

from which we obtain three equilibrium points

$$
\begin{aligned}
& E_{1}=(0,0), \\
& E_{2}=\left(0, \frac{1-\sqrt{\Delta_{1}}}{2 C_{2}}\right), \\
& E_{3}=\left(0, \frac{1+\sqrt{\Delta_{1}}}{2 C_{2}}\right),
\end{aligned}
$$

where $\Delta_{1}=1-4 A_{2} C_{2}$.

Assume that $\bar{x} \neq 0$. Then, from the first equation of system (12) we have

$$
\bar{y}=-\frac{A_{1}+B_{1} \bar{x}-1}{C_{1}} .
$$

By substituting this into the second equation we obtain

$$
A_{1}+B_{1} \bar{x}-1=0
$$

or

$$
\begin{aligned}
\tilde{g}(x) \equiv & B_{1}^{2} C_{2} x^{2} \\
& +x\left(B_{1}\left(2\left(A_{1}-1\right) C_{2}+C_{1}\right)+B_{2} C_{1}^{2}\right) \\
& +\left(A_{1}-1\right)^{2} C_{2}+\left(A_{1}-1\right) C_{1}+A_{2} C_{1}^{2}=0,
\end{aligned}
$$


from which we obtain the other three equilibrium points

$$
\begin{aligned}
E_{4} & =\left(\frac{1-A_{1}}{B_{1}}, 0\right), \\
E_{5} & \\
& =\left(\frac{-2 A_{1} B_{1} C_{2}-B_{2} C_{1}^{2}-B_{1} C_{1}+2 B_{1} C_{2}+C_{1} \sqrt{\Delta_{2}}}{2 B_{1}^{2} C_{2}},\right. \\
& \left.\frac{B_{1}+B_{2} C_{1}-\sqrt{\Delta_{2}}}{2 B_{1} C_{2}}\right), \\
E_{6} & \\
& =\left(\frac{-2 A_{1} B_{1} C_{2}-B_{2} C_{1}^{2}-B_{1} C_{1}+2 B_{1} C_{2}-C_{1} \sqrt{\Delta_{2}}}{2 B_{1}^{2} C_{2}},\right. \\
& \left.\frac{B_{1}+B_{2} C_{1}+\sqrt{\Delta_{2}}}{2 B_{1} C_{2}}\right),
\end{aligned}
$$

where

$$
\Delta_{2}=\left(B_{1}+B_{2} C_{1}\right)^{2}-4 B_{1}\left(A_{2} B_{1}-\left(A_{1}-1\right) B_{2}\right) C_{2} .
$$

Lemma 10. The following hold:

(i) The equilibrium points $E_{2}$ and $E_{3}$ exist if and only if $\Delta_{1} \geq 0$ and $E_{2}=E_{3}$ if and only if $\Delta_{1}=0$.

(ii) The equilibrium point $E_{4}$ exists if and only if $A_{1} \leq 1$ and $E_{4}=E_{1}$ if and only if $A_{1}=1$.

(iii) Assume that $\Delta_{2} \geq 0$. The equilibrium point $E_{5}$ exists if and only if $A_{1}<1$ and

$$
\begin{aligned}
& C_{1} \leq \frac{\left(1-A_{1}\right) B_{1}}{\left(1-A_{1}\right) B_{2}+2 A_{2} B_{1}}, \\
& C_{2} \leq \frac{\left(B_{2} C_{1}+B_{1}\right)^{2}}{4 B_{1}\left(A_{2} B_{1}+\left(1-A_{1}\right) B_{2}\right)}
\end{aligned}
$$

or

$$
\begin{aligned}
& C_{1}>\frac{\left(1-A_{1}\right) B_{1}}{\left(1-A_{1}\right) B_{2}+2 A_{2} B_{1}}, \\
& C_{2} \leq \frac{C_{1}\left(1-A_{1}-A_{2} C_{1}\right)}{\left(1-A_{1}\right)^{2}} .
\end{aligned}
$$

(iv) Assume that $\Delta_{2} \geq 0$. The equilibrium point $E_{6}$ exists if and only if $A_{1}<1$ and

$$
\begin{aligned}
C_{1} & \leq \frac{\left(1-A_{1}\right) B_{1}}{\left(1-A_{1}\right) B_{2}+2 A_{2} B_{1}}, \\
\frac{C_{1}\left(1-A_{1}-A_{2} C_{1}\right)}{\left(1-A_{1}\right)^{2}} & \leq C_{2} \\
& \leq \frac{\left(B_{2} C_{1}+B_{1}\right)^{2}}{4 B_{1}\left(A_{2} B_{1}+\left(1-A_{1}\right) B_{2}\right)} .
\end{aligned}
$$

Proof. The proof of the statements (i) and (ii) is trivial and we skip it. Now we prove the statement (iii). In view of Descartes' rule of signs we obtain that (17) has no positive solutions if $A_{1} \geq 1$. Now, we suppose that $A_{1}<1$. One can see that $\bar{y}_{5}>0$ for all values of parameters. We consider two cases:

(1) Assume that

$$
-2 A_{1} B_{1} C_{2}-B_{2} C_{1}^{2}-B_{1} C_{1}+2 B_{1} C_{2} \geq 0,
$$

which is equivalent to

$$
C_{2} \geq \frac{C_{1}\left(B_{2} C_{1}+B_{1}\right)}{2\left(1-A_{1}\right) B_{1}} .
$$

Since

$$
\begin{aligned}
& \Delta_{2} \geq 0 \Longleftrightarrow \\
& C_{2} \leq \frac{\left(B_{2} C_{1}+B_{1}\right)^{2}}{4 B_{1}\left(A_{2} B_{1}+\left(1-A_{1}\right) B_{2}\right)}
\end{aligned}
$$

we have that $\bar{x}_{5} \geq 0$ if and only if

$$
\begin{aligned}
& \frac{C_{1}\left(B_{2} C_{1}+B_{1}\right)}{2\left(1-A_{1}\right) B_{1}} \leq C_{2} \leq \frac{\left(B_{2} C_{1}+B_{1}\right)^{2}}{4 B_{1}\left(A_{2} B_{1}+\left(1-A_{1}\right) B_{2}\right)}, \\
& \frac{\left(B_{2} C_{1}+B_{1}\right)^{2}}{4 B_{1}\left(A_{2} B_{1}-\left(A_{1}-1\right) B_{2}\right)}-\frac{C_{1}\left(B_{2} C_{1}+B_{1}\right)}{2\left(1-A_{1}\right) B_{1}} \\
& =-\frac{\left(B_{2} C_{1}+B_{1}\right)\left(\left(1-A_{1}\right) B_{2} C_{1}+B_{1}\left(2 A_{2} C_{1}+A_{1}-1\right)\right)}{4\left(1-A_{1}\right) B_{1}\left(A_{2} B_{1}+\left(1-A_{1}\right) B_{2}\right)} \\
& \geq 0
\end{aligned}
$$

which is equivalent to

$$
C_{1} \leq \frac{\left(1-A_{1}\right) B_{1}}{2 A_{2} B_{1}+\left(1-A_{1}\right) B_{2}} .
$$

From (27) and (28) it follows $\bar{x}_{5} \geq 0$ if and only if

$$
C_{1} \leq \frac{\left(1-A_{1}\right) B_{1}}{2 A_{2} B_{1}+\left(1-A_{1}\right) B_{2}},
$$

$\frac{C_{1}\left(B_{2} C_{1}+B_{1}\right)}{2\left(1-A_{1}\right) B_{1}} \leq C_{2} \leq \frac{\left(B_{2} C_{1}+B_{1}\right)^{2}}{4 B_{1}\left(A_{2} B_{1}+\left(1-A_{1}\right) B_{2}\right)}$.

(2) Assume that

$$
-2 A_{1} B_{1} C_{2}-B_{2} C_{1}^{2}-B_{1} C_{1}+2 B_{1} C_{2}<0
$$

which is equivalent to

$$
C_{2}<\frac{C_{1}\left(B_{2} C_{1}+B_{1}\right)}{2\left(1-A_{1}\right) B_{1}}
$$



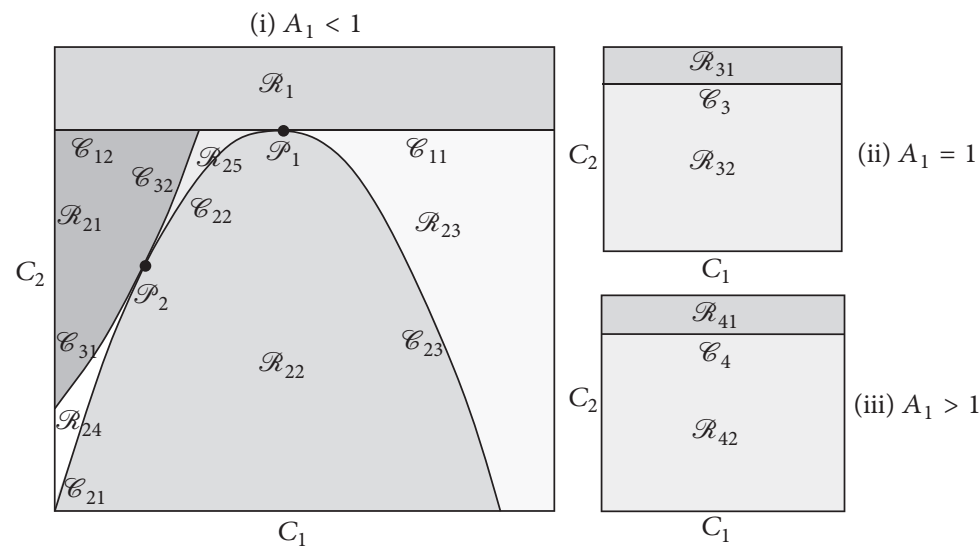

Figure 1: Parameter regions in the $\left(C_{1}, C_{2}\right)$-plane. The curves $\mathscr{C}_{21}, C_{22}$, and $\mathscr{C}_{23}$ are defined as part of the parabola $C_{2}=C_{1}\left(1-A_{1}-A_{2} C_{1}\right) /(1-$ $\left.A_{1}\right)^{2}$ and the curves $\mathscr{C}_{32}$ and $\mathscr{C}_{31}$ are defined as part of the parabola $C_{2}=\left(B_{2} C_{1}+B_{1}\right)^{2} /\left(4 B_{1}\left(A_{2} B_{1}+\left(1-A_{1}\right) B_{2}\right)\right)$.

Then $\bar{x}_{5} \geq 0$ if and only if

$$
\begin{aligned}
& \Delta_{2} C_{1}^{2}-\left(-B_{1}\left(2\left(A_{1}-1\right) C_{2}+C_{1}\right)-B_{2} C_{1}^{2}\right)^{2} \\
& \quad=-4 B_{1}^{2} C_{2}\left(\left(A_{1}-1\right)^{2} C_{2}+C_{1}\left(A_{2} C_{1}+A_{1}-1\right)\right) \\
& \quad \geq 0
\end{aligned}
$$

which is equivalent to

$$
\begin{aligned}
& C_{2} \leq \frac{C_{1}\left(-A_{2} C_{1}-A_{1}+1\right)}{\left(1-A_{1}\right)^{2}}, \\
& \Delta_{2} \geq 0 \Longleftrightarrow \\
& C_{2} \leq \frac{\left(B_{2} C_{1}+B_{1}\right)^{2}}{4 B_{1}\left(A_{2} B_{1}+\left(1-A_{1}\right) B_{2}\right)} .
\end{aligned}
$$

Since

$$
\begin{gathered}
\frac{\left(B_{2} C_{1}+B_{1}\right)^{2}}{4 B_{1}\left(A_{2} B_{1}-\left(A_{1}-1\right) B_{2}\right)}-\frac{C_{1}\left(-A_{2} C_{1}-A_{1}+1\right)}{\left(1-A_{1}\right)^{2}} \\
=\frac{\left(\left(1-A_{1}\right) B_{2} C_{1}+B_{1}\left(-2 A_{2} C_{1}-A_{1}+1\right)\right)^{2}}{4\left(1-A_{1}\right)^{2} B_{1}\left(A_{2} B_{1}+\left(1-A_{1}\right) B_{2}\right)} \geq 0
\end{gathered}
$$

then from (33) and $\Delta_{2} \geq 0$ we have

$$
C_{2} \leq \frac{C_{1}\left(1-A_{2} C_{1}-A_{1}\right)}{\left(1-A_{1}\right)^{2}} .
$$

Since

$$
\begin{aligned}
& \frac{C_{1}\left(1-A_{2} C_{1}-A_{1}\right)}{\left(1-A_{1}\right)^{2}}-\frac{C_{1}\left(B_{2} C_{1}+B_{1}\right)}{2\left(1-A_{1}\right) B_{1}} \\
& =-\frac{C_{1}\left(\left(1-A_{1}\right) B_{2} C_{1}+B_{1}\left(2 A_{2} C_{1}+A_{1}-1\right)\right)}{2\left(A_{1}-1\right)^{2} B_{1}}
\end{aligned}
$$

we have that (31) and (36) are equivalent to

$$
\begin{aligned}
& C_{1}>\frac{\left(1-A_{1}\right) B_{1}}{2 A_{2} B_{1}+\left(1-A_{1}\right) B_{2}}, \\
& C_{2} \leq \frac{C_{1}\left(1-A_{2} C_{1}-A_{1}\right)}{\left(1-A_{1}\right)^{2}}
\end{aligned}
$$

or

$$
\begin{aligned}
& C_{1} \leq \frac{\left(1-A_{1}\right) B_{1}}{2 A_{2} B_{1}+\left(1-A_{1}\right) B_{2}}, \\
& C_{2}<\frac{C_{1}\left(B_{2} C_{1}+B_{1}\right)}{2\left(1-A_{1}\right) B_{1}} .
\end{aligned}
$$

Now, the proof of the statement (iii) follows from (28), (38), and (39). The proof of the statement (iv) is similar and we skip it.

We now introduce the following notation for regions in parameter space $\left(C_{1}, C_{2}\right)$ (see Figure 1):

$$
\begin{aligned}
\mathscr{R}_{24} & =\left\{\left(C_{1}, C_{2}\right): A_{1}<1, \Delta_{1}>0, C_{1}\right. \\
& <\frac{\left(1-A_{1}\right) B_{1}}{\left(1-A_{1}\right) B_{2}+2 A_{2} B_{1}}, \frac{C_{1}\left(1-A_{1}-A_{2} C_{1}\right)}{\left(1-A_{1}\right)^{2}} \\
& \left.<C_{2}<\frac{\left(B_{2} C_{1}+B_{1}\right)^{2}}{4 B_{1}\left(A_{2} B_{1}+\left(1-A_{1}\right) B_{2}\right)}\right\}, \\
\mathscr{R}_{22} & =\left\{\left(C_{1}, C_{2}\right): A_{1}<1, \Delta_{1}>0, C_{2}\right. \\
< & \left.\frac{C_{1}\left(1-A_{1}-A_{2} C_{1}\right)}{\left(1-A_{1}\right)^{2}}\right\}, \\
\mathscr{R}_{21} & =\left\{\left(C_{1}, C_{2}\right): A_{1}<1, \Delta_{1}>0, C_{2}\right. \\
> & \left.\frac{\left(B_{2} C_{1}+B_{1}\right)^{2}}{4 B_{1}\left(A_{2} B_{1}+\left(1-A_{1}\right) B_{2}\right)}\right\},
\end{aligned}
$$




$$
\begin{aligned}
& \mathscr{R}_{25}=\left\{\left(C_{1}, C_{2}\right): A_{1}<1, \Delta_{1}>0, \frac{1-A_{1}}{2 A_{2}}>C_{1}\right. \\
& >\frac{\left(1-A_{1}\right) B_{1}}{\left(1-A_{1}\right) B_{2}+2 A_{2} B_{1}}, \frac{C_{1}\left(1-A_{1}-A_{2} C_{1}\right)}{\left(1-A_{1}\right)^{2}} \\
& \left.<C_{2}<\frac{\left(B_{2} C_{1}+B_{1}\right)^{2}}{4 B_{1}\left(A_{2} B_{1}+\left(1-A_{1}\right) B_{2}\right)}\right\}, \\
& \mathscr{R}_{23}=\left\{\left(C_{1}, C_{2}\right): A_{1}<1, \Delta_{1}>0, C_{1}\right. \\
& >\frac{1-A_{1}}{2 A_{2}}, \frac{C_{1}\left(1-A_{1}-A_{2} C_{1}\right)}{\left(1-A_{1}\right)^{2}}<C_{2} \\
& \left.<\frac{\left(B_{2} C_{1}+B_{1}\right)^{2}}{4 B_{1}\left(A_{2} B_{1}+\left(1-A_{1}\right) B_{2}\right)}\right\} \text {, } \\
& \mathscr{R}_{1}=\left\{\left(C_{1}, C_{2}\right): A_{1}<1, \Delta_{1}<0\right\}, \\
& \mathscr{C}_{31}=\left\{\left(C_{1}, C_{2}\right): A_{1}<1, \Delta_{1}>0, C_{1}\right. \\
& <\frac{\left(1-A_{1}\right) B_{1}}{\left(1-A_{1}\right) B_{2}+2 A_{2} B_{1}}, C_{2} \\
& \left.=\frac{\left(B_{2} C_{1}+B_{1}\right)^{2}}{4 B_{1}\left(A_{2} B_{1}+\left(1-A_{1}\right) B_{2}\right)}\right\}, \\
& \mathscr{C}_{32}=\left\{\left(C_{1}, C_{2}\right): A_{1}<1, \Delta_{1}>0, \frac{1-A_{1}}{2 A_{2}}>C_{1}\right. \\
& >\frac{\left(1-A_{1}\right) B_{1}}{\left(1-A_{1}\right) B_{2}+2 A_{2} B_{1}}, C_{2} \\
& \left.=\frac{\left(B_{2} C_{1}+B_{1}\right)^{2}}{4 B_{1}\left(A_{2} B_{1}+\left(1-A_{1}\right) B_{2}\right)}\right\} \text {, } \\
& \mathscr{C}_{21}=\left\{\left(C_{1}, C_{2}\right): A_{1}<1, \Delta_{1}>0, C_{1}\right. \\
& <\frac{\left(1-A_{1}\right) B_{1}}{\left(1-A_{1}\right) B_{2}+2 A_{2} B_{1}}, C_{2} \\
& \left.=\frac{C_{1}\left(1-A_{1}-A_{2} C_{1}\right)}{\left(1-A_{1}\right)^{2}}\right\} \text {, } \\
& \mathscr{C}_{22}=\left\{\left(C_{1}, C_{2}\right): A_{1}<1, \Delta_{1}>0, \frac{1-A_{1}}{2 A_{2}}>C_{1}\right. \\
& >\frac{\left(1-A_{1}\right) B_{1}}{\left(1-A_{1}\right) B_{2}+2 A_{2} B_{1}}, C_{2} \\
& \left.=\frac{C_{1}\left(1-A_{1}-A_{2} C_{1}\right)}{\left(1-A_{1}\right)^{2}}\right\} \text {, }
\end{aligned}
$$$$
\mathscr{C}_{23}=\left\{\left(C_{1}, C_{2}\right): A_{1}<1, \Delta_{1}>0, C_{1}\right.
$$$$
\begin{aligned}
> & \left.\frac{1-A_{1}}{2 A_{2}}, C_{2}=\frac{C_{1}\left(1-A_{1}-A_{2} C_{1}\right)}{\left(1-A_{1}\right)^{2}}\right\}, \\
\mathscr{C}_{11} & =\left\{\left(C_{1}, C_{2}\right): A_{1}<1, \Delta_{1}=0, C_{1}>\frac{1-A_{1}}{2 A_{2}}\right\}, \\
\mathscr{C}_{12} & =\left\{\left(C_{1}, C_{2}\right): A_{1}<1, \Delta_{1}=0, C_{1}<\frac{1-A_{1}}{2 A_{2}}\right\}, \\
\mathscr{P}_{1} & =\left\{\left(C_{1}, C_{2}\right): A_{1}<1, C_{1}=\frac{1-A_{1}}{2 A_{2}}, C_{2}=\frac{1}{4 A_{2}}\right\}, \\
\mathscr{P}_{2} & =\left\{\left(C_{1}, C_{2}\right): A_{1}<1, C_{1}\right. \\
& =\frac{\left(1-A_{1}\right) B_{1}}{\left(1-A_{1}\right) B_{2}+2 A_{2} B_{1}}, C_{2} \\
= & \left.\frac{B_{1}\left(A_{2} B_{1}-\left(A_{1}-1\right) B_{2}\right)}{\left(\left(A_{1}-1\right) B_{2}-2 A_{2} B_{1}\right)^{2}}\right\}, \\
\mathscr{R}_{31} & =\left\{\left(C_{1}, C_{2}\right): A_{1}=1, \Delta_{1}<0\right\}, \\
\mathscr{R}_{32} & =\left\{\left(C_{1}, C_{2}\right): A_{1}=1, \Delta_{1}>0\right\}, \\
\mathscr{C}_{3}= & \left\{\left(C_{1}, C_{2}\right): A_{1}=1, \Delta_{1}=0\right\}, \\
\mathscr{R}_{41} & =\left\{\left(C_{1}, C_{2}\right): A_{1}>1, \Delta_{1}<0\right\}, \\
\mathscr{R}_{42} & =\left\{\left(C_{1}, C_{2}\right): A_{1}>1, \Delta_{1}>0\right\}, \\
\mathscr{C}_{4}= & \left\{\left(C_{1}, C_{2}\right): A_{1}>1, \Delta_{1}=0\right\} .
\end{aligned}
$$

Figure 1 gives a graphical representation of above sets. The following result gives a complete classification for the number of equilibrium solutions of system (1).

Proposition 11. Let $A_{1}, A_{2}, B_{1}, B_{2}, C_{1}$, and $C_{2}$ be positive real numbers. Then, the number of positive equilibrium solutions of system (1) with parameters $A_{1}, A_{2}, B_{1}, B_{2}, C_{1}$, and $C_{2}$ can be from 1 to 6. The different cases are given in Table 1.

Proof. The proof follows from Lemma 10.

\section{Linearized Stability Analysis}

The Jacobian matrix of the map $T$ has the form

$J_{T}$

$$
=\left(\begin{array}{cc}
\frac{A_{1}+y C_{1}}{\left(A_{1}+x B_{1}+y C_{1}\right)^{2}} & -\frac{x C_{1}}{\left(A_{1}+x B_{1}+y C_{1}\right)^{2}} \\
-\frac{y^{2} B_{2}}{\left(A_{2}+x B_{2}+y^{2} C_{2}\right)^{2}} & \frac{2 y\left(A_{2}+x B_{2}\right)}{\left(A_{2}+x B_{2}+y^{2} C_{2}\right)^{2}}
\end{array}\right) .
$$


TABLE 1: The criteria for the existence of the equilibrium points.

\begin{tabular}{|c|c|c|c|}
\hline Case & Equilibria & Region & The criteria for the existence \\
\hline (i) & $E_{1}, E_{2}, E_{3}, E_{4}, E_{5}, E_{6}$ & $\mathscr{R}_{24}$ & $\begin{array}{c}A_{1}<1, \Delta_{1}>0, C_{1}<\frac{\left(1-A_{1}\right) B_{1}}{\left(1-A_{1}\right) B_{2}+2 A_{2} B_{1}}, \\
\frac{C_{1}\left(1-A_{1}-A_{2} C_{1}\right)}{\left(1-A_{1}\right)^{2}}<C_{2}<\frac{\left(B_{2} C_{1}+B_{1}\right)^{2}}{4 B_{1}\left(A_{2} B_{1}+\left(1-A_{1}\right) B_{2}\right)}\end{array}$ \\
\hline (ii) & $E_{1}, E_{2}, E_{3}, E_{4}, E_{5}=E_{6}$ & $\mathscr{C}_{31}$ & $\begin{array}{c}A_{1}<1, \Delta_{1}>0, C_{1}<\frac{\left(1-A_{1}\right) B_{1}}{\left(1-A_{1}\right) B_{2}+2 A_{2} B_{1}}, \\
C_{2}=\frac{\left(B_{2} C_{1}+B_{1}\right)^{2}}{4 B_{1}\left(A_{2} B_{1}+\left(1-A_{1}\right) B_{2}\right)}\end{array}$ \\
\hline (iii) & $E_{1}, E_{2}, E_{3}, E_{4}, E_{5}$ & $\mathscr{R}_{22}$ & $A_{1}<1, \Delta_{1}>0, C_{2}<\frac{C_{1}\left(1-A_{1}-A_{2} C_{1}\right)}{\left(1-A_{1}\right)^{2}}$ \\
\hline (iv) & $E_{1}, E_{2}, E_{3}, E_{4}$ & $\mathscr{R}_{21} \cup \mathscr{R}_{25} \cup \mathscr{C}_{32}$ & $\begin{array}{c}A_{1}<1, \Delta_{1}>0, C_{2}>\frac{\left(B_{2} C_{1}+B_{1}\right)^{2}}{4 B_{1}\left(A_{2} B_{1}+\left(1-A_{1}\right) B_{2}\right)} \text { or } \\
A_{1}<1, \Delta_{1}>0, \frac{1-A_{1}}{2 A_{2}}>C_{1}>\frac{\left(1-A_{1}\right) B_{1}}{\left(1-A_{1}\right) B_{2}+2 A_{2} B_{1}}, \\
\frac{C_{1}\left(1-A_{1}-A_{2} C_{1}\right)}{\left(1-A_{1}\right)^{2}}<C_{2} \leq \frac{\left(B_{2} C_{1}+B_{1}\right)^{2}}{4 B_{1}\left(A_{2} B_{1}+\left(1-A_{1}\right) B_{2}\right)}\end{array}$ \\
\hline (v) & $E_{1}, E_{2}, E_{3}, E_{4}$ & $\mathscr{R}_{23}$ & $\begin{array}{c}A_{1}<1, \Delta_{1}>0, C_{1}>\frac{1-A_{1}}{2 A_{2}} \\
\frac{C_{1}\left(1-A_{1}-A_{2} C_{1}\right)}{\left(1-A_{1}\right)^{2}}<C_{2} \leq \frac{\left(B_{2} C_{1}+B_{1}\right)^{2}}{4 B_{1}\left(A_{2} B_{1}+\left(1-A_{1}\right) B_{2}\right)}\end{array}$ \\
\hline (vi) & $E_{1}, E_{2}, E_{3}=E_{6}, E_{4}, E_{5}$ & $\mathscr{C}_{21}$ & $A_{1}<1, \Delta_{1}>0, C_{1}<\frac{\left(1-A_{1}\right) B_{1}}{\left(1-A_{1}\right) B_{2}+2 A_{2} B_{1}}, C_{2}=\frac{C_{1}\left(1-A_{1}-A_{2} C_{1}\right)}{\left(1-A_{1}\right)^{2}}$ \\
\hline (vii) & $E_{1}, E_{2}, E_{3}=E_{5}=E_{6}, E_{4}$ & $\mathscr{P}_{2}$ & $A_{1}<1, \Delta_{1}>0, C_{1}=\frac{\left(1-A_{1}\right) B_{1}}{\left(1-A_{1}\right) B_{2}+2 A_{2} B_{1}}, C_{2}=\frac{C_{1}\left(1-A_{1}-A_{2} C_{1}\right)}{\left(1-A_{1}\right)^{2}}$ \\
\hline (viii) & $E_{1}, E_{2}, E_{3}=E_{5}, E_{4}$ & $\mathscr{C}_{22}$ & $\begin{array}{c}A_{1}<1, \Delta_{1}>0, \frac{1-A_{1}}{2 A_{2}}>C_{1}>\frac{\left(1-A_{1}\right) B_{1}}{\left(1-A_{1}\right) B_{2}+2 A_{2} B_{1}} \\
C_{2}=\frac{C_{1}\left(1-A_{1}-A_{2} C_{1}\right)}{\left(1-A_{1}\right)^{2}}\end{array}$ \\
\hline (ix) & $E_{1}, E_{3}, E_{2}=E_{5}, E_{4}$ & $\mathscr{C}_{23}$ & $A_{1}<1, \Delta_{1}>0, C_{1}>\frac{1-A_{1}}{2 A_{2}}, C_{2}=\frac{C_{1}\left(1-A_{1}-A_{2} C_{1}\right)}{\left(1-A_{1}\right)^{2}}$ \\
\hline (x) & $E_{1}, E_{2}=E_{3}=E_{5}, E_{4}$ & $\mathscr{P}_{1}$ & $A_{1}<1, \Delta_{1}=0, C_{1}=\frac{1-A_{1}}{2 A_{2}}, C_{2}=\frac{C_{1}\left(1-A_{1}-A_{2} C_{1}\right)}{\left(1-A_{1}\right)^{2}}$ \\
\hline (xi) & $E_{1}, E_{2}=E_{3}, E_{4}$ & $\mathscr{C}_{11} \cup \mathscr{C}_{12}$ & $A_{1}<1, \Delta_{1}=0, C_{1} \neq \frac{1-A_{1}}{2 A_{2}}$ \\
\hline (xii) & $E_{1}, E_{4}$ & $\mathscr{R}_{1}$ & $A_{1}<1, \Delta_{1}<0$ \\
\hline (xiii) & $E_{1}=E_{4}, E_{2}, E_{3}$ & $\mathscr{R}_{32}$ & $A_{1}=1, \Delta_{1}>0$ \\
\hline (xiv) & $E_{1}=E_{4}, E_{2}=E_{3}$ & $\mathscr{C}_{3}$ & $A_{1}=1, \Delta_{1}=0$ \\
\hline$(\mathrm{xv})$ & $E_{1}=E_{4}$ & $\mathscr{R}_{31}$ & $A_{1}=1, \Delta_{1}<0$ \\
\hline (xvi) & $E_{1}, E_{2}, E_{3}$ & $\mathscr{R}_{42}$ & $A_{1}>1, \Delta_{1}>0$ \\
\hline (xvii) & $E_{1}, E_{2}=E_{3}$ & $\mathscr{C}_{4}$ & $A_{1}>1, \Delta_{1}=0$ \\
\hline (xviii) & $E_{1}$ & $\mathscr{R}_{41}$ & $A_{1}>1, \Delta_{1}<0$ \\
\hline
\end{tabular}

The determinant of (41) at the equilibrium point is given by

$$
\begin{aligned}
\operatorname{det} & J_{T}(\bar{x}, \bar{y}) \\
= & \frac{\bar{y}\left(2 A_{1}\left(A_{2}+B_{2} \bar{x}\right)+\bar{y}\left(2 A_{2}+B_{2} \bar{x}\right) C_{1}\right)}{\left(A_{1}+B_{1} \bar{x}+C_{1} \bar{y}\right)^{2}\left(A_{2}+B_{2} \bar{x}+C_{2} \bar{y}^{2}\right)^{2}}
\end{aligned}
$$

and the trace of (41) at the equilibrium point is given by

$$
\begin{aligned}
\operatorname{tr} J_{T}(\bar{x}, \bar{y})= & \frac{A_{1}+\bar{y} C_{1}}{\left(A_{1}+B_{1} \bar{x}+C_{1} \bar{y}\right)^{2}} \\
& +\frac{2 \bar{y}\left(A_{2}+B_{2} \bar{x}\right)}{\left(A_{2}+B_{2} \bar{x}+C_{2} \bar{y}^{2}\right)^{2}} .
\end{aligned}
$$


The characteristic equation has the form

$$
\begin{aligned}
& \lambda^{2} \\
& -\lambda\left(\frac{A_{1}+\bar{y} C_{1}}{\left(A_{1}+B_{1} \bar{x}+C_{1} \bar{y}\right)^{2}}+\frac{2 \bar{y}\left(A_{2}+B_{2} \bar{x}\right)}{\left(A_{2}+B_{2} \bar{x}+C_{2} \bar{y}^{2}\right)^{2}}\right) \\
& +\frac{\bar{y}\left(2 A_{1}\left(A_{2}+B_{2} \bar{x}\right)+\bar{y}\left(2 A_{2}+B_{2} \bar{x}\right) C_{1}\right)}{\left(A_{1}+B_{1} \bar{x}+C_{1} \bar{y}\right)^{2}\left(A_{2}+B_{2} \bar{x}+C_{2} \bar{y}^{2}\right)^{2}}=0 .
\end{aligned}
$$

Lemma 12. The following statements hold:

(a) $E_{1}$ is locally asymptotically stable if $A_{1}>1$.

(b) $E_{1}$ is a saddle point if $A_{1}<1$.

(c) $E_{1}$ is a nonhyperbolic equilibrium point if $A_{1}=1$.

Proof. We have that, for the equilibrium point $E_{1}, \operatorname{tr} J_{T}\left(E_{1}\right)=$ $1 / A_{1}$ and $\operatorname{det} J_{T}\left(E_{1}\right)=0$. The characteristic equation of (50) at $E_{1}$ has the form $\lambda^{2}-\left(1 / A_{1}\right) \lambda=0$, from which the proof follows.

Lemma 13. The following statements hold:

(a) $E_{4}$ is locally asymptotically stable if $A_{1}<1$.

(b) $E_{4}$ is a nonhyperbolic equilibrium point if $A_{1}=1$.

Proof. We have that, for the equilibrium point $E_{4}, \operatorname{tr} J_{T}\left(E_{4}\right)=$ $A_{1}$ and $\operatorname{det} J_{T}\left(E_{4}\right)=0$. The characteristic equation of $(50)$ at $E_{4}$ has the form $\lambda^{2}-A_{1} \lambda=0$, from which the proof follows.

The equilibrium points $E_{5}$ and $E_{6}$ are intersection points of the curves

$$
\begin{aligned}
& x_{f}(y)=\frac{1-A_{1}-C_{1} y}{B_{1}}, \\
& x_{g}(y)=\frac{y-A_{2}-C_{2} y^{2}}{B_{2}} .
\end{aligned}
$$

Let $\tilde{x}(y)=x_{f}(y)-x_{g}(y)$ for $y \in\left[\left(1-\sqrt{1-4 A_{2} C_{2}}\right) / 2 C_{2},(1+\right.$ $\left.\left.\sqrt{1-4 A_{2} C_{2}}\right) / 2 C_{2}\right]$.

Lemma 14. Let $T=(f, g)$ be the map defined by (11). Then $f_{x}^{\prime}\left(E_{5}\right)<1, f_{x}^{\prime}\left(E_{6}\right)<1, g_{y}^{\prime}\left(E_{6}\right)<1$. Let

$$
\begin{aligned}
\tilde{f}(y)= & B_{1} C_{2} y^{2}-\left(B_{2} C_{1}+B_{1}\right) y+A_{2} B_{1} \\
& +B_{2}\left(1-A_{1}\right) .
\end{aligned}
$$

Then, $\bar{y}_{5}$ and $\bar{y}_{6}$ are zeros of $\tilde{f}(y)$ and $\operatorname{sign}(\tilde{x}(y))=\operatorname{sign}(\tilde{f}(y))$ for $y \in\left[\left(1-\sqrt{1-4 A_{2} C_{2}}\right) / 2 C_{2},\left(1+\sqrt{1-4 A_{2} C_{2}}\right) / 2 C_{2}\right]$.

Proof. The first derivative of $x_{f}\left(\bar{y}_{6}\right)$ is given by

$$
x_{f}^{\prime}\left(\bar{y}_{i}\right)=\frac{f_{y}^{\prime}\left(E_{i}\right)}{1-f_{x}^{\prime}\left(E_{i}\right)}=-\frac{C_{1}}{B_{1}}<0, \quad i=5,6 .
$$

Since $f_{y}^{\prime}\left(E_{i}\right)<0, i=5,6$, we get $f_{x}^{\prime}\left(E_{i}\right)<1, i=5,6$. Similarly, one can see that

$$
\begin{aligned}
& x_{g}^{\prime}\left(\bar{y}_{6}\right)=\frac{1-g_{y}^{\prime}\left(E_{6}\right)}{g_{x}^{\prime}\left(E_{6}\right)} \\
& =-\frac{\sqrt{\left(B_{2} C_{1}+B_{1}\right)^{2}-4 B_{1} C_{2}\left(A_{2} B_{1}-\left(A_{1}-1\right) B_{2}\right)}+B_{2} C_{1}}{B_{1} B_{2}} \\
& <0 .
\end{aligned}
$$

Since $g_{x}^{\prime}\left(E_{6}\right)<0$, we get $g_{y}^{\prime}\left(E_{6}\right)<1$. Further,

$$
\begin{aligned}
\tilde{x}(y) & =\frac{1-A_{1}-C_{1} y}{B_{1}}-\frac{y-A_{2}-C_{2} y^{2}}{B_{2}} \\
= & \frac{B_{1} C_{2} y^{2}-\left(B_{2} C_{1}+B_{1}\right) y+A_{2} B_{1}-A_{1} B_{2}+B_{2}}{B_{1} B_{2}} \\
= & \frac{\tilde{f}(y)}{B_{1} B_{2}},
\end{aligned}
$$

from which the proof follows.

Lemma 15. Let $T$ be the map associated with system (1) and

$$
J_{T}\left(\bar{x}_{i}, \bar{y}_{i}\right)=\left(\begin{array}{ll}
a_{i} & b_{i} \\
c_{i} & d_{i}
\end{array}\right)
$$

be the Jacobian matrix of $T$ at fixed point $E_{i}(i=5,6)$. Then the Jacobian matrix (50) has real and distinct eigenvalues $\lambda_{1}$ and $\lambda_{2}$ such that $0<\lambda_{1} \leq \lambda_{2}$. Furthermore, the following hold:

$$
\begin{aligned}
& \operatorname{sign}\left(\tilde{x}^{\prime}\left(\bar{y}_{6}\right)\right)=\operatorname{sign}\left(1-\lambda_{2}^{(6)}\right), \quad \lambda_{2}^{(6)}<1, \\
& \operatorname{sign}\left(\tilde{x}^{\prime}\left(\bar{y}_{5}\right)\right)=\operatorname{sign}\left(1-\lambda_{2}^{(5)}\right)\left(1-\lambda_{2}^{(5)}\right) .
\end{aligned}
$$

Proof. Implicit differentiation of the equations defining $C_{f}$ and $C_{g}$ at $E_{i}$ gives

$$
\begin{aligned}
& x_{f}^{\prime}\left(\bar{y}_{i}\right)=\frac{f_{y}^{\prime}\left(E_{i}\right)}{1-f_{x}^{\prime}\left(E_{i}\right)}, \\
& x_{g}^{\prime}\left(\bar{y}_{i}\right)=\frac{1-g_{y}^{\prime}\left(E_{i}\right)}{g_{x}^{\prime}\left(E_{i}\right)} .
\end{aligned}
$$

Characteristic equations associated with the Jacobian matrix of $T$ at $E_{i}$ are given by

$$
\begin{aligned}
p(\lambda)= & \lambda^{2}-\left[f_{x}^{\prime}\left(E_{i}\right)+g_{y}^{\prime}\left(E_{i}\right)\right] \lambda \\
& +\left[f_{x}^{\prime}\left(E_{i}\right) g_{y}^{\prime}\left(E_{i}\right)-f_{y}^{\prime}\left(E_{i}\right) g_{x}^{\prime}\left(E_{i}\right)\right] \\
= & \lambda^{2}-\left(a_{i}+d_{i}\right) \lambda+\left(a_{i} d_{i}-b_{i} c_{i}\right) .
\end{aligned}
$$


Since the map $T$ is competitive, then the eigenvalues of the Jacobian matrix of the map $T$, at the equilibrium $E_{i}$, are real and distinct and furthermore $0<\lambda_{1}^{(i)}<\lambda_{2}^{(i)}$. By (53), we have

$$
\begin{aligned}
\tilde{x}^{\prime}\left(\bar{y}_{i}\right) & =x_{f}^{\prime}\left(\bar{y}_{i}\right)-x_{g}^{\prime}\left(\bar{y}_{i}\right) \\
& =\frac{f_{y}^{\prime}\left(E_{i}\right)}{1-f_{x}^{\prime}\left(E_{i}\right)}-\frac{1-g_{y}^{\prime}\left(E_{i}\right)}{g_{x}^{\prime}\left(E_{i}\right)} \\
& =\frac{b_{i}}{1-a_{i}}-\frac{1-d_{i}}{c_{i}} \\
& =\frac{-1+\left(a_{i}+d_{i}\right)-\left(a_{i} d_{i}-b_{i} c_{i}\right)}{c_{i}\left(1-a_{i}\right)}=\frac{-p(1)}{c_{i}\left(1-a_{i}\right)} \\
& =\frac{\left(1-\lambda_{1}^{(i)}\right)\left(1-\lambda_{2}^{(i)}\right)}{c_{i}\left(a_{i}-1\right)} .
\end{aligned}
$$

In view of Lemma 14 and from $\operatorname{tr} J_{T}\left(E_{6}\right)=a_{6}+d_{6}=f_{x}^{\prime}\left(E_{6}\right)+$ $g_{y}^{\prime}\left(E_{6}\right)=\lambda_{1}^{(6)}+\lambda_{2}^{(6)}<2$ we get $\lambda_{1}^{(6)}<1$. The map $T$ is competitive, which implies $c_{6}=g_{x}^{\prime}\left(E_{6}\right)<0$. In view of Lemma 14 we get $a_{6}=f_{x}^{\prime}\left(E_{6}\right)<1$ from which it follows (51). Similarly, from $c_{5}=g_{x}^{\prime}\left(E_{5}\right)<0$ and $a_{5}=f_{x}^{\prime}\left(E_{5}\right)<1$ we obtain (52).

The following lemma describes the local stability of the equilibrium points $E_{5}$ and $E_{6}$.

Lemma 16. Assume that $A_{1}<1$ and $\Delta_{2} \geq 0$. Then the following hold:

(i) If $\Delta_{2}>0$ and $E_{6}$ exists then it is locally asymptotically stable.

(ii) If $\Delta_{2}>0$ and $E_{5}$ exists then it is a saddle point.

(iii) If $\Delta_{2}=0$ then $E_{5}=E_{6}$. Furthermore, if $E_{5}=E_{6}$ exists then it is nonhyperbolic equilibrium point. The eigenvalues of $J_{T}\left(E_{5}=E_{6}\right)$ are given by $\lambda_{1}=1$ and $\lambda_{2}<1$.

Proof. Assuming that $\Delta_{2}>0$, then $\bar{y}_{5}$ and $\bar{y}_{6}$ are zeros of multiplicity one of

$$
\begin{aligned}
\tilde{f}(y)= & B_{1} C_{2} y^{2}-\left(B_{2} C_{1}+B_{1}\right) y+A_{2} B_{1} \\
& +B_{2}\left(1-A_{1}\right)
\end{aligned}
$$

and $\bar{y}_{6}>\bar{y}_{5}>0$. From this we have $\tilde{f}(y)>0$ for $y \in\left(0, \bar{y}_{5}\right) \cup$ $\left(\bar{y}_{6},+\infty\right)$ and $\tilde{f}(y)<0$ for $y \in\left(\bar{y}_{5}, \bar{y}_{5}\right)$.

By Lemmas 6 and 7 from [19] the equilibrium curves $x_{f}(y)$ and $x_{g}(y)$ intersect transversally at $E_{5}$ and $E_{6}$, that is, $\tilde{x}^{\prime}\left(\bar{y}_{i}\right) \neq 0 i=5,6$. By this and Lemma 14 and by continuity of function $\tilde{x}(y)$ there exists a neighborhood $U_{\bar{y}_{i}}^{(i)}$ of $\bar{y}_{i}$ such that $\tilde{x}^{\prime}(y)>0$ for $y \in U_{\bar{y}_{6}}$ and $\tilde{x}^{\prime}(y)<0$ for $y \in U_{\bar{y}_{5}}^{(5)}$. This implies that $\tilde{x}^{\prime}\left(\bar{y}_{6}\right)>0$ and $\tilde{x}^{\prime}\left(\bar{y}_{5}\right)<0$. By Lemma 15 we have that $E_{6}$ is locally asymptotically stable and $E_{5}$ is a saddle point whenever equilibrium points $E_{5}$ and $E_{6}$ exist.
Assume that $\Delta_{2}=0$. Then $\bar{y}_{5}=\bar{y}_{6}$ is zero of $\tilde{f}(y)$ of multiplicity two. In view of Lemmas 6 and 7 from [19] we have that $\tilde{x}^{\prime}\left(\bar{y}_{5}\right)=0$. The rest of the proof follows from the proof of Lemma 15.

Lemma 17. Assume that $\Delta_{1} \geq 0$. The following statements are true:

(a) $E_{2}$ is a saddle point if $\Delta_{1}>0$, and $A_{1} \geq 1$ or $A_{1}<1$ and $C_{1}>\left(1-A_{1}\right)\left(1+\sqrt{1-4 A_{2} C_{2}}\right) / 2 A_{2}$.

(b) $E_{2}$ is a repeller if $\Delta_{1}>0, A_{1}<1$, and $C_{1}<\left(1-A_{1}\right)(1+$ $\left.\sqrt{1-4 A_{2} C_{2}}\right) / 2 A_{2}$.

(c) $E_{2}$ is a nonhyperbolic equilibrium point if $\Delta_{1}=0$ or

$$
\begin{aligned}
& \Delta_{1}>0, \\
& A_{1}<1, \\
& C_{1}=\frac{\left(1-A_{1}\right)\left(1+\sqrt{1-4 A_{2} C_{2}}\right)}{2 A_{2}} .
\end{aligned}
$$

If $\Delta_{1}=0$ then the eigenvalues of $J_{T}\left(E_{2}\right)$ are given by

$$
\begin{aligned}
& \lambda_{1}=1, \\
& \lambda_{2}=\frac{1}{2 A_{2} C_{1}+A_{1}}
\end{aligned}
$$

with corresponding eigenvectors

$$
\begin{aligned}
& \mathbf{v}_{1}=(0,1)^{T}, \\
& \mathbf{v}_{2}=\left(\frac{2 A_{2} C_{1}+A_{1}-1}{B_{2}\left(2 A_{2} C_{1}+A_{1}\right)}, 1\right)^{T} .
\end{aligned}
$$

If (57) holds then the eigenvalues of $J_{T}\left(E_{2}\right)$ are given by

$$
\begin{aligned}
& \lambda_{1}=1, \\
& \lambda_{2}=1+\sqrt{1-4 A_{2} C_{2}}>1
\end{aligned}
$$

with corresponding eigenvectors

$$
\begin{aligned}
& \mathbf{v}_{1}=\left(\frac{\sqrt{1-4 A_{2} C_{2}}}{B_{2}}, 1\right)^{T}, \\
& \mathbf{v}_{2}=(0,1)^{T} .
\end{aligned}
$$

Proof. One can see that

$$
\begin{aligned}
& 1+\operatorname{det} J_{T}\left(E_{2}\right)-\operatorname{tr} J_{T}\left(E_{2}\right) \\
& =\frac{2\left(1-A_{1}\right) \sqrt{1-4 A_{2} C_{2}} C_{2}+C_{1}\left(1-4 A_{2} C_{2}-\sqrt{1-4 A_{2} C_{2}}\right)}{2 A_{1} C_{2}+C_{1}\left(1-\sqrt{1-4 A_{2} C_{2}}\right)}, \\
& 1-\operatorname{det} J_{T}\left(E_{2}\right) \\
& =\frac{C_{1}\left(1-\sqrt{1-4 A_{2} C_{2}}\right)+2 C_{2}\left(A_{1}-1-\sqrt{1-4 A_{2} C_{2}}\right)}{2 A_{1} C_{2}+C_{1}\left(1-\sqrt{1-4 A_{2} C_{2}}\right)} .
\end{aligned}
$$


(a) Since det $J_{T}\left(E_{2}\right)>0$ and $\operatorname{tr} J_{T}\left(E_{2}\right)>0$, the equilibrium $E_{2}$ is a saddle point if and only if $1+\operatorname{det} J_{T}\left(E_{2}\right)-$ $\operatorname{tr} J_{T}\left(E_{2}\right)<0$. If $A_{1} \geq 1$ it is obvious that $1+$ $\operatorname{det} J_{T}\left(E_{2}\right)-\operatorname{tr} J_{T}\left(E_{2}\right)<0$. Assume that $A_{1}<1$. Then $1+\operatorname{det} J_{T}\left(E_{2}\right)-\operatorname{tr} J_{T}\left(E_{2}\right)<0$ if and only if

$$
\begin{aligned}
C_{1}> & \frac{2\left(1-A_{1}\right) C_{2} \sqrt{1-4 A_{2} C_{2}}}{4 A_{2} C_{2}-1+\sqrt{1-4 A_{2} C_{2}}} \\
& =\frac{\left(1-A_{1}\right)\left(\sqrt{1-4 A_{2} C_{2}}+1\right)}{2 A_{2}},
\end{aligned}
$$

from which the proof of the statement follows.

(b) Since $\operatorname{det} J_{T}\left(E_{2}\right)>0$ and $\operatorname{tr} J_{T}\left(E_{2}\right)>0$, the equilibrium $E_{2}$ is repeller if and only if $1+\operatorname{det} J_{T}\left(E_{2}\right)-$ $\operatorname{tr} J_{T}\left(E_{2}\right)>0$ and $1-\operatorname{det} J_{T}\left(E_{2}\right)<0$. The proof of the statement follows from the facts

$$
\begin{aligned}
& 1-\operatorname{det} J_{T}\left(E_{2}\right)<0 \Longleftrightarrow \\
& C_{1}<\frac{2 C_{2}\left(\sqrt{1-4 A_{2} C_{2}}-A_{1}+1\right)}{1-\sqrt{1-4 A_{2} C_{2}}}, \\
& \frac{2\left(1-A_{1}\right) C_{2} \sqrt{1-4 A_{2} C_{2}}}{4 A_{2} C_{2}-1+\sqrt{1-4 A_{2} C_{2}}} \\
& -\frac{2 C_{2}\left(\sqrt{1-4 A_{2} C_{2}}-A_{1}+1\right)}{1-\sqrt{1-4 A_{2} C_{2}}} \\
& =-\frac{-4 A_{2} C_{2}+\sqrt{1-4 A_{2} C_{2}}+1}{2 A_{2}}<0 .
\end{aligned}
$$

(c) Since det $J_{T}\left(E_{2}\right)>0$ and $\operatorname{tr} J_{T}\left(E_{2}\right)>0$, the equilibrium $E_{2}$ is nonhyperbolic if and only if $1+\operatorname{det} J_{T}\left(E_{2}\right)-$ $\operatorname{tr} J_{T}\left(E_{2}\right)=0$ or $\operatorname{det} J_{T}\left(E_{2}\right)=1$ and $\operatorname{tr} J_{T}\left(E_{2}\right) \leq 2$. From the proof of the statements (a) and (b) if

$$
\begin{aligned}
\Delta_{1} & =0 \\
\text { or } \Delta_{1} & >0, \\
A_{1} & <1, \\
C_{1} & =\frac{\left(1-A_{1}\right)\left(\sqrt{1-4 A_{2} C_{2}}+1\right)}{2 A_{2}}
\end{aligned}
$$

we obtain $1+\operatorname{det} J_{T}\left(E_{2}\right)-\operatorname{tr} J_{T}\left(E_{2}\right)=0$. Now, assume that $\Delta_{1}>0$ and

$$
\begin{aligned}
\operatorname{det} J_{T}\left(E_{2}\right) & =1 \Longleftrightarrow \\
C_{1} & =\frac{2 C_{2}\left(\sqrt{1-4 A_{2} C_{2}}-A_{1}+1\right)}{1-\sqrt{1-4 A_{2} C_{2}}} .
\end{aligned}
$$

This implies $\operatorname{tr} J_{T}\left(E_{2}\right)-2=\left(1-4 A_{2} C_{2}\right) /$ $\left(\sqrt{1-4 A_{2} C_{2}}+1\right)>0$. The rest of the proof follows from the fact that if $\Delta_{1}=0$ then

$$
J_{T}\left(E_{2}\right)=\left(\begin{array}{cc}
\frac{1}{A_{1}+2 A_{2} C_{1}} & 0 \\
0 & 1
\end{array}\right)
$$

and if (57) holds then

$$
J_{T}\left(E_{2}\right)=\left(\begin{array}{cc}
1 & 0 \\
0 & \sqrt{1-4 A_{2} C_{2}}+1
\end{array}\right) .
$$

Lemma 18. Assume that $\Delta_{1} \geq 0$. The following statements are true:

(a) $E_{3}$ is locally asymptotically stable if $\Delta_{1}>0$, and $A_{1} \geq 1$ or $A_{1}<1$ and $C_{1}>\left(1-A_{1}\right)\left(1-\sqrt{1-4 A_{2} C_{2}}\right) / 2 A_{2}$.

(b) $E_{3}$ is a saddle point if $\Delta_{1}>0$, and $A_{1}<1, C_{1}<$ $\left(1-A_{1}\right)\left(1-\sqrt{1-4 A_{2} C_{2}}\right) / 2 A_{2}$.

(c) $E_{3}$ is a nonhyperbolic equilibrium point if $\Delta_{1}=0$ or

$$
\begin{aligned}
& \Delta_{1}>0, \\
& A_{1}<1, \\
& C_{1}=\frac{\left(1-A_{1}\right)\left(1-\sqrt{1-4 A_{2} C_{2}}\right)}{2 A_{2}} .
\end{aligned}
$$

If $\Delta_{1}=0$ then the eigenvalues of $J_{T}\left(E_{3}\right)$ are given by

$$
\begin{aligned}
& \lambda_{1}=1, \\
& \lambda_{2}=\frac{1}{2 A_{2} C_{1}+A_{1}}
\end{aligned}
$$

with corresponding eigenvectors

$$
\begin{aligned}
& \mathbf{v}_{1}=(0,1)^{T}, \\
& \mathbf{v}_{2}=\left(\frac{2 A_{2} C_{1}+A_{1}-1}{B_{2}\left(2 A_{2} C_{1}+A_{1}\right)}, 1\right)^{T} .
\end{aligned}
$$

If (57) holds then the eigenvalues of $J_{T}\left(E_{3}\right)$ are given by

$$
\begin{aligned}
& \lambda_{1}=1, \\
& \lambda_{2}=1-\sqrt{1-4 A_{2} C_{2}}<1
\end{aligned}
$$

with corresponding eigenvectors

$$
\begin{aligned}
& \mathbf{v}_{1}=\left(-\frac{\sqrt{1-4 A_{2} C_{2}}}{B_{2}}, 1\right)^{T} \\
& \mathbf{v}_{2}=(0,1)^{T} .
\end{aligned}
$$

Proof. Since the proof of this lemma is similar to the proof of Lemma 17, it is omitted.

We summarize results about local stability in the following theorem.

Theorem 19. Let $A_{1}, A_{2}, B_{1}, B_{2}, C_{1}$, and $C_{2}$ be positive real numbers. Then, local stability of the equilibrium points for different parameter regions is given by Table 2.

Proof. The proof follows from Theorem 8 and Lemmas 17 and 18.

Figure 2 illustrates visually local stability of all equilibrium points of system (1). 
TABLE 2: The local stability of the equilibrium points.

\begin{tabular}{lll}
\hline Case & The local stability & Parameter region \\
\hline
\end{tabular}

(i)

Saddle: $E_{1}, E_{3}, E_{5}$; LAS: $E_{4}, E_{6}$; repeller: $E_{2}$

$$
\begin{gathered}
A_{1}<1, \Delta_{1}>0, C_{1}<\frac{\left(1-A_{1}\right) B_{1}}{\left(1-A_{1}\right) B_{2}+2 A_{2} B_{1}}, \\
\frac{C_{1}\left(1-A_{1}-A_{2} C_{1}\right)}{\left(1-A_{1}\right)^{2}}<C_{2}<\frac{\left(B_{2} C_{1}+B_{1}\right)^{2}}{4 B_{1}\left(A_{2} B_{1}+\left(1-A_{1}\right) B_{2}\right)}
\end{gathered}
$$

(ii)

Saddle: $E_{1}, E_{3}$; LAS: $E_{4}$;

nonhyperbolic: $E_{5}=E_{6}$; repeller: $E_{2}$

(iii)

Saddle: $E_{1}, E_{5}$; LAS: $E_{3}, E_{4}$; repeller: $E_{2}$

$$
A_{1}<1, \Delta_{1}>0, C_{1}<\frac{\left(1-A_{1}\right) B_{1}}{\left(1-A_{1}\right) B_{2}+2 A_{2} B_{1}}, C_{2}=\frac{\left(B_{2} C_{1}+B_{1}\right)^{2}}{4 B_{1}\left(A_{2} B_{1}+\left(1-A_{1}\right) B_{2}\right)}
$$
reller: $E_{2}$

$$
A_{1}<1, \Delta_{1}>0, C_{2}<\frac{C_{1}\left(1-A_{1}-A_{2} C_{1}\right)}{\left(1-A_{1}\right)^{2}}
$$

$$
A_{1}<1, \Delta_{1}>0, C_{2}>\frac{\left(B_{2} C_{1}+B_{1}\right)^{2}}{4 B_{1}\left(A_{2} B_{1}+\left(1-A_{1}\right) B_{2}\right)} \text { or } A_{1}<1, \Delta_{1}>0 \text {, }
$$

(iv) Saddle: $E_{1}, E_{3}$; LAS: $E_{4}$; repeller: $E_{2}$

$$
\begin{gathered}
\frac{1-A_{1}}{2 A_{2}}>C_{1}>\frac{\left(1-A_{1}\right) B_{1}}{\left(1-A_{1}\right) B_{2}+2 A_{2} B_{1}}, \\
\frac{C_{1}\left(1-A_{1}-A_{2} C_{1}\right)}{\left(1-A_{1}\right)^{2}}<C_{2} \leq \frac{\left(B_{2} C_{1}+B_{1}\right)^{2}}{4 B_{1}\left(A_{2} B_{1}+\left(1-A_{1}\right) B_{2}\right)}
\end{gathered}
$$

(v) Saddle: $E_{1}, E_{2}$; LAS: $E_{3}, E_{4}$

$$
A_{1}<1, \Delta_{1}>0, C_{1}>\frac{1-A_{1}}{2 A_{2}}, \frac{C_{1}\left(1-A_{1}-A_{2} C_{1}\right)}{\left(1-A_{1}\right)^{2}}<C_{2} \leq \frac{\left(B_{2} C_{1}+B_{1}\right)^{2}}{4 B_{1}\left(A_{2} B_{1}+\left(1-A_{1}\right) B_{2}\right)}
$$

Saddle: $E_{1}, E_{5}$; LAS: $E_{4}$; repeller: $E_{2}$

$$
\begin{aligned}
A_{1}<1, \Delta_{1}>0, C_{1}<\frac{\left(1-A_{1}\right) B_{1}}{\left(1-A_{1}\right) B_{2}+2 A_{2} B_{1}}, \\
C_{2}=\frac{C_{1}\left(1-A_{1}-A_{2} C_{1}\right)}{\left(1-A_{1}\right)^{2}}
\end{aligned}
$$

(vi)

Nonhyperbolic: $E_{3}=E_{6}$;

(vii)

Saddle: $E_{1}$; LAS: $E_{4}$; repeller:

$$
E_{2}
$$

$$
A_{1}<1, \Delta_{1}>0, C_{1}=\frac{\left(1-A_{1}\right) B_{1}}{\left(1-A_{1}\right) B_{2}+2 A_{2} B_{1}}, C_{2}=\frac{C_{1}\left(1-A_{1}-A_{2} C_{1}\right)}{\left(1-A_{1}\right)^{2}}
$$

Nonhyperbolic: $E_{3}=E_{5}=E_{6}$;

\begin{tabular}{|c|c|c|}
\hline (xi) & $\begin{array}{c}\text { Saddle: } E_{1} ; \text { LAS: } E_{4} ; \\
\text { nonhyperbolic: } E_{2}=E_{3}\end{array}$ & $A_{1}<1, \Delta_{1}=0, C_{1} \neq \frac{1-A_{1}}{2 A_{2}}$ \\
\hline (xii) & Saddle: $E_{1} ;$ LAS: $E_{4}$ & $A_{1}<1, \Delta_{1}<0$ \\
\hline (xiii) & $\begin{array}{c}\text { Saddle: } E_{2} ; \text { LAS: } E_{3} ; \\
\text { nonhyperbolic: } E_{1}=E_{4}\end{array}$ & $A_{1}=1, \Delta_{1}>0$ \\
\hline (xiv) & $\begin{array}{c}\text { Nonhyperbolic: } E_{1}=E_{4} \text {, } \\
E_{2}=E_{3}\end{array}$ & $A_{1}=1, \Delta_{1}=0$ \\
\hline$(\mathrm{xv})$ & Nonhyperbolic: $E_{1}=E_{4}$ & $A_{1}=1, \Delta_{1}<0$ \\
\hline (xvi) & Saddle: $E_{1}, E_{3} ;$ LAS: $E_{2}$ & $A_{1}>1, \Delta_{1}>0$ \\
\hline (xvii) & $\begin{array}{c}\text { LAS: } E_{1} ; \text { nonhyperbolic: } \\
\qquad E_{2}=E_{3}\end{array}$ & $A_{1}>1, \Delta_{1}=0$ \\
\hline (xviii) & LAS: $E_{1}$ & $A_{1}>1, \Delta_{1}<0$ \\
\hline
\end{tabular}

(viii)

Saddle: $E_{1}$; LAS: $E_{4}$; repeller:

Nonhyperbolic: $E_{3}=E_{5}$

(ix)

Saddle: $E_{1}$; LAS: $E_{3}, E_{4}$; nonhyperbolic: $E_{2}=E_{5}$

$$
A_{1}<1, \Delta_{1}>0, \frac{1-A_{1}}{2 A_{2}}>C_{1}>\frac{\left(1-A_{1}\right) B_{1}}{\left(1-A_{1}\right) B_{2}+2 A_{2} B_{1}}, C_{2}=\frac{C_{1}\left(1-A_{1}-A_{2} C_{1}\right)}{\left(1-A_{1}\right)^{2}}
$$

$$
\begin{gathered}
A_{1}<1, \Delta_{1}>0, C_{1}>\frac{1-A_{1}}{2 A_{2}}, \\
C_{2}=\frac{C_{1}\left(1-A_{1}-A_{2} C_{1}\right)}{\left(1-A_{1}\right)^{2}}
\end{gathered}
$$

(x) Saddle: $E_{1}$; LAS: $E_{4}$; nonhyperbolic: $E_{2}=E_{3}=E_{5}$

$$
A_{1}<1, \Delta_{1}=0, C_{1}=\frac{1-A_{1}}{2 A_{2}}, C_{2}=\frac{C_{1}\left(1-A_{1}-A_{2} C_{1}\right)}{\left(1-A_{1}\right)^{2}}
$$




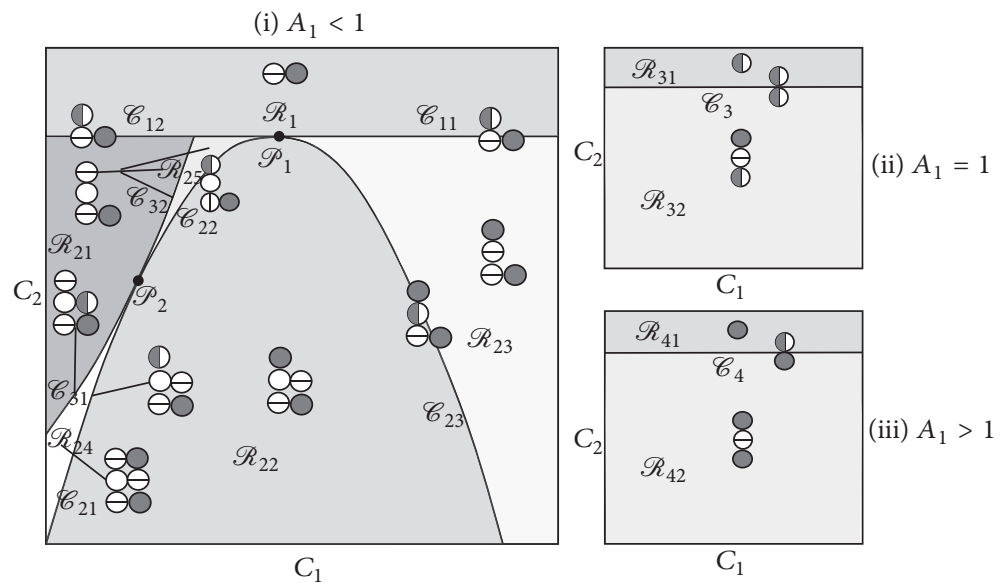

FIGURE 2: Equilibria in different parameter regions for the number of equilibria of system (1) when $A_{1}, A_{2}, B_{1}$, and $B_{2}$ are fixed positive real numbers, as given by Proposition 11. Each circle in the parameters $\left(C_{1}, C_{2}\right)$-plane indicates the existence of an isolated equilibrium point of system (1) in the nonnegative quadrant of the $x y$-plane. Local stability character of equilibria as given in Theorem 19 is indicated as follows: $\bullet$, locally asymptotically stable equilibrium; $\ominus$, saddle; $\bigcirc$, repelling equilibrium point: two-colored circle, semistable nonhyperbolic equilibrium.

\section{Injectivity and Convergence to Equilibrium Points}

In this section we prove some global properties of the map $T$ such as injectivity and $(\mathrm{O}+)$ property and give global behavior on the coordinate axes.

Lemma 20. The map $T$ is injective.

Proof. Assume that $T\left(x_{1}, y_{1}\right)=T\left(x_{2}, y_{2}\right)$. Then, we have

$$
\begin{aligned}
& \left(\frac{A_{1} x_{1}-A_{1} x_{2}-C_{1} x_{2} y_{1}+C_{1} x_{1} y_{2}}{\left(A_{1}+B_{1} x_{1}+C_{1} y_{1}\right)\left(A_{1}+B_{1} x_{2}+C_{1} y_{2}\right)},\right. \\
& \left.\frac{A_{2} y_{1}^{2}+B_{2} x_{2} y_{1}^{2}-A_{2} y_{2}^{2}-B_{2} x_{1} y_{2}^{2}}{\left(A_{2}+B_{2} x_{1}+C_{2} y_{1}^{2}\right)\left(A_{2}+B_{2} x_{2}+C_{2} y_{2}^{2}\right)}\right) \\
& \quad=(0,0) .
\end{aligned}
$$

Equation (74) is equivalent to

$$
\begin{aligned}
A_{1} x_{1}-A_{1} x_{2}-C_{1} x_{2} y_{1}+C_{1} x_{1} y_{2} & =0 \\
A_{2} y_{1}^{2}+B_{2} x_{2} y_{1}^{2}-A_{2} y_{2}^{2}-B_{2} x_{1} y_{2}^{2} & =0
\end{aligned}
$$

Equation (75) implies

$$
x_{1}=\frac{A_{1} x_{2}+C_{1} x_{2} y_{1}}{A_{1}+C_{1} y_{2}}
$$

By substituting this into (76) we obtain

$$
\frac{\left(y_{1}-y_{2}\right)\left(A_{1} A_{2} y_{1}+A_{1} B_{2} x_{2} y_{1}+A_{1} A_{2} y_{2}+A_{1} B_{2} x_{2} y_{2}+A_{2} C_{1} y_{1} y_{2}+B_{2} C_{1} x_{2} y_{1} y_{2}+A_{2} C_{1} y_{2}^{2}\right)}{A_{1}+C_{1} y_{2}}=0,
$$

from which it follows that $y_{1}=y_{2}$. From (77) we have $x_{1}=$ $x_{2}$, which complete the proof.

The global behavior of $T$ on the coordinate axes is described with the following result.

Lemma 21. The following statements hold:

(i) If $\Delta_{1} \geq 0$ and $\left(x_{n}, y_{n}\right)=T^{n}\left(0, y_{0}\right)$ then $x_{n}=0$ and $\left(x_{n}, y_{n}\right) \rightarrow E_{3}$ for $y_{0} \in\left(\left(1-\sqrt{1-4 A_{2} C_{2}}\right) /\right.$ $\left.2 C_{2}, \infty\right)$ and $\left(x_{n}, y_{n}\right) \rightarrow E_{1}$ for $y_{0} \in(0,(1+$ $\left.\left.\sqrt{1-4 A_{2} C_{2}}\right) / 2 C_{2}\right)$. (ii) If $\Delta_{1}<0$ and $\left(x_{n}, y_{n}\right)=T^{n}\left(0, y_{0}\right)$ then $x_{n}=0$ and $\left(x_{n}, y_{n}\right) \rightarrow E_{1}$ for $y_{0} \in(0, \infty)$.

(iii) If $A_{1}<1$ and $\left(x_{n}^{\prime}, y_{n}^{\prime}\right)=T^{n}\left(x_{0}, 0\right)$ then $y_{n}^{\prime}=0$ and $\left(x_{n}^{\prime}, y_{n}^{\prime}\right) \rightarrow E_{4}$ for $x_{0} \in(0, \infty)$.

(iv) If $A_{1} \geq 1$ and $\left(x_{n}^{\prime}, y_{n}^{\prime}\right)=T^{n}\left(x_{0}, 0\right)$ then $y_{n}^{\prime}=0$ and $\left(x_{n}^{\prime}, y_{n}^{\prime}\right) \rightarrow E_{1}$ for $x_{0} \in(0, \infty)$.

(v) $T([0, \infty) \times[0, \infty)) \subset\left[0,1 / B_{1}\right] \times\left[0,1 / C_{2}\right]$. 
Proof.

(i) From (11) it is easy to see that if $x_{0}=0$ then $x_{n}=0$ for $n>0$. Since

$T\left(0, y_{0}\right)-\left(0, y_{0}\right)=\left(0,-\frac{y_{0}\left(C_{2} y_{0}^{2}-y_{0}+A_{2}\right)}{A_{2}+C_{2} y_{0}^{2}}\right)$

we obtain that

$\left(0, y_{0}\right) \preceq_{\mathrm{se}} T\left(0, y_{0}\right)$

for $y_{0} \in\left(0, \frac{1-\sqrt{1-4 A_{2} C_{2}}}{2 C_{2}}\right) \cup\left(\frac{1+\sqrt{1-4 A_{2} C_{2}}}{2 C_{2}}, \infty\right)$,

$T\left(0, y_{0}\right) \preceq_{\text {se }}\left(0, y_{0}\right)$

$$
\text { for } y_{0} \in\left(\frac{1-\sqrt{1-4 A_{2} C_{2}}}{2 C_{2}}, \frac{1+\sqrt{1-4 A_{2} C_{2}}}{2 C_{2}}\right) \text {. }
$$

Take $y_{0}>\left(1+\sqrt{1-4 A_{2} C_{2}}\right) / 2 C_{2}$. Then $T^{n}(0$, $\left.y_{0}\right) \preceq_{\text {se }} T^{n+1}\left(0, y_{0}\right) \preceq_{\text {se }} E_{3}$. Since $T^{n}\left(0, y_{0}\right) \preceq_{\text {se }} E_{3} \prec_{\text {se }}$ $E_{2} \prec$ se $E_{1}$ we obtain $T^{n}\left(0, y_{0}\right) \rightarrow E_{3}$ as $n \rightarrow \infty$. Similarly, if $y_{0} \in\left(\left(1-\sqrt{1-4 A_{2} C_{2}}\right) / 2 C_{2}\right.$, $\left.\left(1+\sqrt{1-4 A_{2} C_{2}}\right) / 2 C_{2}\right)$ then $E_{3} \preceq_{\mathrm{se}} T^{n+1}\left(0, y_{0}\right) \preceq_{\text {se }}$ $T^{n}\left(0, y_{0}\right) \preceq_{\mathrm{se}} E_{2} \prec_{\mathrm{se}} E_{1}$ which implies $T^{n}\left(0, y_{0}\right) \rightarrow E_{3}$ as $n \rightarrow \infty$. If $y_{0}<\left(1-\sqrt{1-4 A_{2} C_{2}}\right) / 2 C_{2}$ then $E_{3} \prec_{\mathrm{se}} E_{2} \prec_{\mathrm{se}} T^{n}\left(0, y_{0}\right) \preceq_{\mathrm{se}} T^{n+1}\left(0, y_{0}\right) \preceq_{\mathrm{se}} E_{1} \quad$ which implies $T^{n}\left(0, y_{0}\right) \rightarrow E_{1}$ as $n \rightarrow \infty$.

(ii) If $\Delta_{1}<0$ then $T$ has only equilibrium $E_{1}$ on $y$ axis and $T\left(0, y_{0}\right) \preceq_{\text {se }}\left(0, y_{0}\right)$ for all $y_{0} \geq 0$. Since $T$ is monotone map we get $T^{n}\left(0, y_{0}\right) \preceq_{\mathrm{se}} T^{n+1}\left(0, y_{0}\right) \preceq_{\mathrm{se}} E_{1}$ which implies $T^{n}\left(0, y_{0}\right) \rightarrow E_{1}$ as $n \rightarrow \infty$ from which the proof follows.

(iii) The proof of the statements (iii) and (iv) is similar to the proof of statements (i) and (ii) and follows from the fact that

$T\left(x_{0}, 0\right)-\left(x_{0}, 0\right)=\left(-\frac{x_{0}\left(A_{1}+B_{1} x_{0}-1\right)}{A_{1}+B_{1} x_{0}}, 0\right)$

and will be omitted.

(v) The proof follows from the facts that $f(x, y) \leq 1 / B_{1}$ and $g(x, y) \leq 1 / C_{2}$.

Lemma 22. Let $M(t) \equiv\left(t,\left(1-A_{1}-B_{1} t\right) / C_{1}\right)$. Then $M\left(\bar{x}_{6}\right)=$ $E_{6}, M\left(\bar{x}_{5}\right)=E_{5}$, and $M\left(\bar{x}_{4}\right)=E_{4}$ and the following hold:

(i) If $\Delta_{2} \geq 0$ then $M(t) \preceq_{s e} T(M(t))$ for $t \in\left(0, \bar{x}_{6}\right) \cup$ $\left(\bar{x}_{5}, \bar{x}_{4}\right)$ and $T(M(t)) \preceq_{s e} M(t)$ for $t \in\left(\bar{x}_{6}, \bar{x}_{5}\right)$.

(ii) If $\Delta_{2}<0$ then $M(t) \preceq_{s e} T(M(t))$ for $t \in\left(0, \bar{x}_{4}\right)$ and $T(M(t)) \preceq_{s e} M(t)$ for $t \in\left(\bar{x}_{6}, \bar{x}_{5}\right)$.

Proof. The proof follows from the fact

$$
\begin{aligned}
T & (M(t))-M(t) \\
& =\left(0, \frac{\left(A_{1}+B_{1} t-1\right) \widetilde{g}(x)}{C_{1}^{3}\left(A_{2}+B_{2} t\right)+C_{2} C_{1}\left(A_{1}+B_{1} t-1\right)^{2}}\right),
\end{aligned}
$$

where $\widetilde{g}(x)$ is given by (17) and $\widetilde{g}\left(\bar{x}_{5}\right)=\widetilde{g}\left(\bar{x}_{6}\right)=0$ and $\bar{x}_{4}=$ $\left(1-A_{1}\right) / B_{1}$.
Theorem 23. Every solution of system (1) converges to an equilibrium point.

Proof. The map $T$ associated with the system is injective. Relation (42) implies that determinant of Jacobian (41) is positive for all $x \in[0, \infty) \times[0, \infty)$. By using Lemma 20 we have that condition $(\mathrm{O}+)$ of Theorem 3 is satisfied for the map $T$ ( $T$ is competitive). Theorem 2 implies that $T^{n}(x)$ is eventually componentwise monotone for all $x \in[0, \infty)^{2}$. The statement (v) of Lemma 21 implies that every solution enters in compact set $\left[0,1 / B_{1}\right] \times\left[0,1 / C_{2}\right]$, from which the proof follows.

Remark 24. In view of Theorem 23 the main objective in determining the global dynamics of system (1) is to characterize the basins of attractions of all equilibrium points. As we will see in Theorem 25 the boundaries of these basins of attractions will be the global stable manifolds of the saddle or nonhyperbolic equilibrium points, whose existence is guaranteed by Theorems 7, 8, and 9 .

\section{Global Behavior}

In this section we give results which precisely describe global dynamics of system (1) including precise characterization of basins of attraction of different equilibrium points. The main result of this paper is the following.

Theorem 25. The global behavior of system (1) is given by Table 3. See Figure 3 for visual illustration of dynamic scenarios.

Proof. We will prove statements (i)-(x) listed in the second column of Table 3 in the given order. The proof of other statements is similar. Let $\mathscr{R}=[0, \infty) \times[0, \infty)$.

(i) Suppose $\left(C_{1}, C_{2}\right) \in \mathscr{R}_{24}$. By Proposition 11, in $Q_{1}(0,0)$ there exist six equilibria $E_{1}, E_{2}, E_{3}, E_{4}, E_{5}$, and $E_{6}$. By Theorem 19 equilibria $E_{4}$ and $E_{5}$ are locally asymptotically stable; $E_{3}, E_{4}$, and $E_{5}$ are the saddle points and $E_{2}$ is repeller. In view of (41) the map $T$ is competitive on $\mathscr{R}$ and strongly competitive on $\operatorname{int}(\mathscr{R})$. It follows from the Perron-Frobenius Theorem and a change of variables [16] that, at each point, the Jacobian matrix of a strongly competitive map has two real and distinct eigenvalues, the larger one in absolute value being positive, and that corresponding eigenvectors may be chosen to point in the direction of the second and first quadrant, respectively. Also, one can show that if the map is strongly competitive then no eigenvector is aligned with a coordinate axis. Hence, all conditions of Theorems 7, 8, and 9 are satisfied, which yields the existence of the global stable manifold $\mathscr{W}^{s}\left(E_{5}\right)$, with endpoint at point $E_{2}$, which is graph of an increasing function. Let $\mathscr{W}^{-}=\{(x, y)$ | $(x, y) \preceq_{\text {se }}\left(\tilde{x}_{0}, \tilde{y}_{0}\right)$ for some $\left.\left(\tilde{x}_{0}, \tilde{y}_{0}\right) \in \mathscr{W}^{s}\left(E_{5}\right)\right\}$ and $\mathscr{W}^{+}=\left\{(x, y) \mid\left(\tilde{x}_{1}, \tilde{y}_{1}\right) \preceq_{\text {se }}(x, y)\right.$ for some $\left(\tilde{x}_{1}, \tilde{y}_{1}\right) \in$ $\left.\mathscr{W}^{s}\left(E_{5}\right)\right\}$. By Lemma 21 and uniqueness of the global stable manifold we have $\mathscr{W}^{\mathcal{s}}\left(E_{1}\right)=\{(0, y): 0 \leq$ 
TABLE 3: The global behavior of system (1).

\begin{tabular}{|c|c|c|}
\hline Case & Parameter region & Global behavior \\
\hline (i) & $\begin{array}{l}\mathscr{R}_{24}: A_{1}<1, \Delta_{1}>0 \\
C_{1}<\frac{\left(1-A_{1}\right) B_{1}}{\left(1-A_{1}\right) B_{2}+2 A_{2} B_{1}} \\
\frac{C_{1}\left(1-A_{1}-A_{2} C_{1}\right)}{\left(1-A_{1}\right)^{2}}<C_{2}< \\
\quad \frac{\left(B_{2} C_{1}+B_{1}\right)^{2}}{4 B_{1}\left(A_{2} B_{1}+\left(1-A_{1}\right) B_{2}\right)}\end{array}$ & $\begin{array}{l}\text { There exist six equilibrium points } E_{1}, E_{2}, E_{3}, E_{4}, E_{5} \text {, and } E_{6} \text {, where } E_{4} \\
\text { and } E_{5} \text { are locally asymptotically stable, } E_{3}, E_{4} \text {, and } E_{6} \text { are saddle } \\
\text { points, and } E_{2} \text { is repeller. The stable manifold } \mathscr{W}^{s}\left(E_{6}\right) \text { of the saddle } \\
\text { point } E_{6} \text { is an increasing separatrix with endpoint at } E_{2} \text {, and solutions } \\
\text { with initial point above the } \mathscr{W}^{s}\left(E_{6}\right) \text { converge to } E_{5} \text {, while solutions } \\
\text { with initial point below the } \mathscr{W}^{s}\left(E_{6}\right) \text { converge to } E_{4} \text {. All orbits that } \\
\text { start on } \mathscr{W}^{s}\left(E_{6}\right) \text { are attracted to } E_{6} \text {. The basins of attraction of } E_{1} \text { and } \\
E_{3} \text { are, respectively, } \\
\mathscr{B}\left(E_{1}\right)=\left\{(0, y): 0 \leq y<\left(1-\sqrt{1-4 A_{2} C_{2}}\right) /\left(2 A_{2}\right)\right\} \text { and } \\
\mathscr{B}\left(E_{3}\right)=\left\{(0, y): y>\left(1-\sqrt{1-4 A_{2} C_{2}}\right) /\left(2 A_{2}\right)\right\}\end{array}$ \\
\hline (ii) & $\begin{array}{c}\mathscr{C}_{31}: A_{1}<1, \Delta_{1}>0, \\
C_{1}<\frac{\left(1-A_{1}\right) B_{1}}{\left(1-A_{1}\right) B_{2}+2 A_{2} B_{1}}, \\
C_{2}=\frac{\left(B_{2} C_{1}+B_{1}\right)^{2}}{4 B_{1}\left(A_{2} B_{1}+\left(1-A_{1}\right) B_{2}\right)}\end{array}$ & $\begin{array}{l}\text { There exist five equilibrium points } E_{1}, E_{2}, E_{3}, E_{4} \text {, and } E_{5}=E_{6} \text {, where } \\
E_{4} \text { is locally asymptotically stable, } E_{1} \text { and } E_{3} \text { are saddle points, } E_{2} \text { is } \\
\text { repeller, and } E_{5}=E_{6} \text { is nonhyperbolic. There exists a continuous } \\
\text { increasing curve } \mathscr{C} \text { with endpoint at } E_{2} \text {, which is a subset of the basin } \\
\text { of attraction of } E_{5}=E_{6} \text {. All solutions with initial point above } \mathscr{C} \\
\text { converge to } E_{5}=E_{6} \text {, while solutions with initial point below } \mathscr{C} \\
\text { converge to } E_{4} \text {. The basins of attraction of } E_{1} \text { and } E_{3} \text { are, respectively, } \\
\mathscr{B}\left(E_{1}\right)=\left\{(0, y): 0 \leq y<\left(1-\sqrt{1-4 A_{2} C_{2}}\right) /\left(2 A_{2}\right)\right\} \text { and } \\
\mathscr{B}\left(E_{3}\right)=\left\{(0, y): y>\left(1-\sqrt{1-4 A_{2} C_{2}}\right) /\left(2 A_{2}\right)\right\}\end{array}$ \\
\hline (iii) & $\begin{array}{c}\mathscr{R}_{22}: A_{1}<1, \Delta_{1}>0, \\
C_{2}<\frac{C_{1}\left(1-A_{1}-A_{2} C_{1}\right)}{\left(1-A_{1}\right)^{2}}\end{array}$ & $\begin{array}{l}\text { There exist five equilibrium points } E_{1}, E_{2}, E_{3}, E_{4} \text {, and } E_{5} \text {, where } E_{3} \\
\text { and } E_{4} \text { are locally asymptotically stable, } E_{1} \text { and } E_{5} \text { are saddle points, } \\
\text { and } E_{2} \text { is repeller. The stable manifold } \mathscr{W}^{s}\left(E_{5}\right) \text { of the saddle point } E_{5} \\
\text { is an increasing separatrix with endpoint at } E_{2} \text {, and solutions with } \\
\text { initial point above } \mathscr{W}^{s}\left(E_{5}\right) \text { converge to } E_{3} \text {, while solutions with initial } \\
\text { point below } \mathscr{W}^{s}\left(E_{5}\right) \text { converge to } E_{4} \text {. All orbits that start on } \mathscr{W}^{s}\left(E_{5}\right) \\
\text { are attracted to } E_{5} \text {. The basin of attraction of } E_{1} \text { is } \\
\mathscr{B}\left(E_{1}\right)=\left\{(0, y): 0 \leq y<\left(1-\sqrt{1-4 A_{2} C_{2}}\right) /\left(2 A_{2}\right)\right\}\end{array}$ \\
\hline
\end{tabular}

$$
\begin{aligned}
& \mathscr{R}_{21} \cup \mathscr{R}_{25} \cup \mathscr{C}_{32}: A_{1}<1, \Delta_{1}>0, \\
& C_{2}>\frac{\left(B_{2} C_{1}+B_{1}\right)^{2}}{4 B_{1}\left(A_{2} B_{1}+\left(1-A_{1}\right) B_{2}\right)} \text { or } A_{1}<1, \\
& \frac{1-A_{1}}{2 A_{2}}>C_{1}>\frac{\left(1-A_{1}\right) B_{1}}{\left(1-A_{1}\right) B_{2}+2 A_{2} B_{1}} \text {, } \\
& \frac{C_{1}\left(1-A_{1}-A_{2} C_{1}\right)}{\left(1-A_{1}\right)^{2}}<C_{2}< \\
& \left(B_{2} C_{1}+B_{1}\right)^{2}
\end{aligned}
$$$$
\overline{4 B_{1}\left(A_{2} B_{1}+\left(1-A_{1}\right) B_{2}\right)}
$$$$
\mathscr{R}_{23}: A_{1}<1, \Delta_{1}>0 \text {, }
$$$$
C_{1}>\frac{\left(1-A_{1}\right) B_{1}}{\left(1-A_{1}\right) B_{2}+2 A_{2} B_{1}} \text {, }
$$$$
\frac{C_{1}\left(1-A_{1}-A_{2} C_{1}\right)}{\left(1-A_{1}\right)^{2}}<C_{2}<
$$$$
\left(B_{2} C_{1}+B_{1}\right)^{2}
$$$$
\overline{4 B_{1}\left(A_{2} B_{1}+\left(1-A_{1}\right) B_{2}\right)}
$$
globally asymptotically stable, $E_{1}$ and $E_{3}$ are saddle points, and $E_{2}$ is a repeller. The basins of attraction of $E_{1}, E_{3}$, and $E_{4}$ are, respectively,

There exist four equilibrium points $E_{1}, E_{2}, E_{3}$, and $E_{4}$, where $E_{3}$ and $E_{4}$ are locally asymptotically stable and $E_{1}$ and $E_{2}$ are saddle points. The stable manifold $\mathscr{W}^{s}\left(E_{2}\right)$ of the saddle point $E_{2}$ is an increasing separatrix, and solutions with initial point above $\mathscr{W}^{s}\left(E_{1}\right)$ converge to $E_{3}$, while solutions with initial point below $\mathscr{W}^{s}\left(E_{2}\right)$ converge to $E_{4}$. All orbits that start on $\mathscr{W}^{s}\left(E_{2}\right)$ are attracted to $E_{2}$. The basin of attraction of $E_{1}$ is given by $\mathscr{B}\left(E_{1}\right)=\left\{(0, y): 0 \leq y<\left(1-\sqrt{1-4 A_{2} C_{2}}\right) /\left(2 A_{2}\right)\right\}$. There exist five equilibrium points $E_{1}, E_{2}, E_{3}=E_{6}, E_{4}$, and $E_{5}$, where $E_{4}$ is locally asymptotically stable, $E_{1}$ and $E_{5}$ are saddle points, $E_{3}=E_{6}$ is nonhyperbolic, and $E_{2}$ is a repeller. The stable manifold $\mathscr{W}^{s}\left(E_{5}\right)$ of the saddle point $E_{5}$ is an increasing separatrix with endpoint at $E_{2}$, and solutions with initial point above $\mathscr{W}^{s}\left(E_{5}\right)$ converge to $E_{3}=E_{6}$, while solutions with initial point below $\mathscr{W}^{s}\left(E_{5}\right)$ converge to $E_{4}$. All orbits that start on $\mathscr{W}^{s}\left(E_{5}\right)$ are attracted to $E_{5}$. The basin of attraction of $E_{1}$ is given by $\mathscr{B}\left(E_{1}\right)=\left\{(0, y): 0 \leq y<\left(1-\sqrt{1-4 A_{2} C_{2}}\right) /\left(2 A_{2}\right)\right\}$.

$$
\begin{gathered}
\mathscr{C}_{21}: A_{1}<1, \Delta_{1}>0, \\
C_{1}<\frac{\left(1-A_{1}\right) B_{1}}{\left(1-A_{1}\right) B_{2}+2 A_{2} B_{1}}, \\
C_{2}=\frac{C_{1}\left(1-A_{1}-A_{2} C_{1}\right)}{\left(1-A_{1}\right)^{2}}
\end{gathered}
$$

There exist four equilibrium points $E_{1}, E_{2}, E_{3}=E_{5}=E_{6}$, and $E_{4}$, where $E_{4}$ is locally asymptotically stable, $E_{1}$ is a saddle point, $E_{3}=E_{5}=E_{6}$ is nonhyperbolic, and $E_{2}$ is a repeller. There exists a strictly increasing curve $\mathscr{C}$ with endpoint at $E_{3}=E_{5}=E_{6}$, which is the subset of the basin of attraction of $E_{3}=E_{5}=E_{6}$. All solutions with initial point above $\mathscr{C}$ converge to $E_{3}=E_{5}=E_{6}$, while solutions with initial point below $\mathscr{C}$ converge to $E_{4}$. The basin of attraction of $E_{1}$ is given by $\mathscr{B}\left(E_{1}\right)=\left\{(0, y): 0 \leq y<\left(1-\sqrt{1-4 A_{2} C_{2}}\right) /\left(2 A_{2}\right)\right\}$. 
TABLE 3: Continued.

\begin{tabular}{cc}
\hline Case & Parameter region \\
\hline $\mathscr{C}_{22}: A_{1}<1, \Delta_{1}>0$, \\
$C_{2}=\frac{C_{1}\left(1-A_{1}-A_{2} C_{1}\right)}{\left(1-A_{1}\right)^{2}}$, \\
$\frac{1-A_{1}}{2 A_{2}}>C_{1}>\frac{\left(1-A_{1}\right) B_{1}}{\left(1-A_{1}\right) B_{2}+2 A_{2} B_{1}}$ \\
\hline
\end{tabular}

(ix)

$$
\begin{gathered}
\mathscr{C}_{23}: A_{1}<1, \Delta_{1}>0, \\
C_{2}=\frac{C_{1}\left(1-A_{1}-A_{2} C_{1}\right)}{\left(1-A_{1}\right)^{2}}, \frac{1-A_{1}}{2 A_{2}}<C_{1}
\end{gathered}
$$

Global behavior

There exist five equilibrium points $E_{1}, E_{2}, E_{3}=E_{5}$, and $E_{4}$, where $E_{4}$ is locally asymptotically stable, $E_{1}$ is a saddle point, $E_{3}=E_{5}$ is nonhyperbolic, and $E_{2}$ is a repeller. The basins of attraction of $E_{1}$ and $E_{3}$ are given by $\mathscr{B}\left(E_{1}\right)=\left\{(0, y): 0 \leq y<\left(1-\sqrt{1-4 A_{2} C_{2}}\right) /\left(2 A_{2}\right)\right\}$ and $\mathscr{B}\left(E_{3}\right)=\left\{(0, y): y>\left(1-\sqrt{1-4 A_{2} C_{2}}\right) /\left(2 A_{2}\right)\right\}$ and the basin of attraction of $E_{4}$ is $\mathscr{B}\left(E_{4}\right)=\{(x, y): x>0, y \geq 0\}$.

There exist four equilibrium points $E_{1}, E_{3}, E_{2}=E_{5}$, and $E_{4}$, where $E_{3}$ and $E_{4}$ are locally asymptotically stable, $E_{1}$ is a saddle point, and $E_{2}=E_{5}$ is nonhyperbolic. There exist continuous increasing curves $\mathscr{C}_{1}$ and $\mathscr{C}_{2}$ with endpoint at $E_{2}=E_{5}$, which are the subsets of the basin of attraction of $E_{2}=E_{5}$. Further, all solutions with initial point above $\mathscr{C}_{2}$ converge to $E_{3}$ and all solutions with initial point above $\mathscr{C}_{1}$ and below $\mathscr{C}_{2}$ converge to $E_{2}$, while solutions with initial point below $\mathscr{C}_{1}$ converge to $E_{4}$. The basin of attraction of $E_{1}$ is $\mathscr{B}\left(E_{1}\right)=\left\{(0, y): 0 \leq y<\left(1-\sqrt{1-4 A_{2} C_{2}}\right) /\left(2 A_{2}\right)\right\}$.

There exist three equilibrium points $E_{1}, E_{2}=E_{3}$, and $E_{4}$, where $E_{4}$ is locally asymptotically stable, $E_{1}$ is a saddle point, and $E_{2}=E_{3}$ is nonhyperbolic. There exists a continuous increasing curve $\mathscr{C}$ with

(x) $\quad A_{1}<1, \Delta_{1}=0, C_{1} \neq \frac{1-A_{1}}{2 A_{2}}$ endpoint at $E_{2}=E_{3}$ which is a subset of the basin of attraction of $E_{2}=E_{3}$. All solutions with initial point above $\mathscr{C}$ converge to $E_{2}=E_{3}$, while solutions with initial point below $\mathscr{C}$ converge to $E_{4}$. The basin of attraction of $E_{1}$ is given by $\mathscr{B}\left(E_{1}\right)=\left\{(0, y): 0 \leq y<\left(1-\sqrt{1-4 A_{2} C_{2}}\right) /\left(2 A_{2}\right)\right\}$.

(xi) $\quad A_{1}<1, \Delta_{1}<0$ There exist two equilibrium points $E_{1}$, which is a saddle point, and $E_{4}$, which is globally asymptotically stable, where $\mathscr{B}\left(E_{4}\right)=(0, \infty) \times[0, \infty)$ and $\mathscr{B}\left(E_{1}\right)=\{(0, y): y \geq 0\}$.

There exist three equilibrium points $E_{1}=E_{4}, E_{2}$, and $E_{3}$, where $E_{1}=E_{4}$ is nonhyperbolic, $E_{3}$ is locally asymptotically stable, and $E_{2}$ is a saddle point. The stable manifold $\mathscr{W}^{s}\left(E_{2}\right)$ of the saddle point $E_{2}$ is

$$
A_{1}=1, \Delta_{1}>0
$$
an increasing separatrix with endpoint at $E_{2}$, and solutions with initial point above $\mathscr{W}^{s}\left(E_{2}\right)$ converge to $E_{3}$, while solutions with initial point below $\mathscr{W}^{s}\left(E_{2}\right)$ converge to $E_{4}$. All orbits that start on $\mathscr{W}^{s}\left(E_{2}\right)$ are attracted to $E_{2}$. The basin of attraction of $E_{1}$ is given by $\mathscr{B}\left(E_{1}\right)=\left\{(0, y): 0 \leq y<\left(1-\sqrt{1-4 A_{2} C_{2}}\right) /\left(2 A_{2}\right)\right\}$.

There exist two equilibrium points $E_{1}=E_{4}$ and $E_{2}=E_{3}$ which are nonhyperbolic. There exists a continuous increasing curve $\mathscr{C}$ with

$$
A_{1}=1, \Delta_{1}=0
$$
endpoint at $E_{2}=E_{3}$ which is a subset of the basin of attraction of $E_{2}=E_{3}$. All solutions with initial point above $\mathscr{C}$ converge to $E_{2}=E_{3}$, while solutions with initial point below $\mathscr{C}$ converge to $E_{1}=E_{4}$. There exists one equilibrium point $E_{1}=E_{4}$ which is nonhyperbolic and global attractor. The basin of attraction of $E_{1}$ is

(xiv)

$$
A_{1}=1, \Delta_{1}<0
$$
$\mathscr{B}\left(E_{1}\right)=[0, \infty) \times[0, \infty)$

There exist three equilibrium points $E_{1}, E_{2}$, and $E_{3}$, where $E_{1}$ and $E_{3}$ are locally asymptotically stable and $E_{2}$ is a saddle point. The stable manifold $\mathscr{W}^{s}\left(E_{2}\right)$ of the saddle point $E_{2}$ is an increasing separatrix with endpoint at $E_{2}$, and solutions with initial point above $\mathscr{W}^{s}\left(E_{2}\right)$ converge to $E_{3}$, while solutions with initial point below $\mathscr{W}^{s}\left(E_{2}\right)$ converge to $E_{1}$. All orbits that start on $\mathscr{W}^{s}\left(E_{2}\right)$ are attracted to $E_{2}$.

$$
A_{1}>1, \Delta_{1}>0
$$

There exists one equilibrium point $E_{1}$ which is globally asymptotically stable. The basin of attraction of $E_{1}$ is $\mathscr{B}\left(E_{1}\right)=[0, \infty) \times[0, \infty)$

There exist two equilibrium points $E_{1}$ and $E_{2}=E_{3}$, where $E_{1}$ is locally asymptotically stable and $E_{2}=E_{3}$ is nonhyperbolic. There exists a continuous increasing curve $\mathscr{C}$ with endpoint at $E_{2}=E_{3}$ which is a subset of the basin of attraction of $E_{2}=E_{3}$. All solutions with initial point above $\mathscr{C}$ converge to $E_{2}=E_{3}$, while solutions with initial point below $\mathscr{C}$ converge to $E_{1}$. 

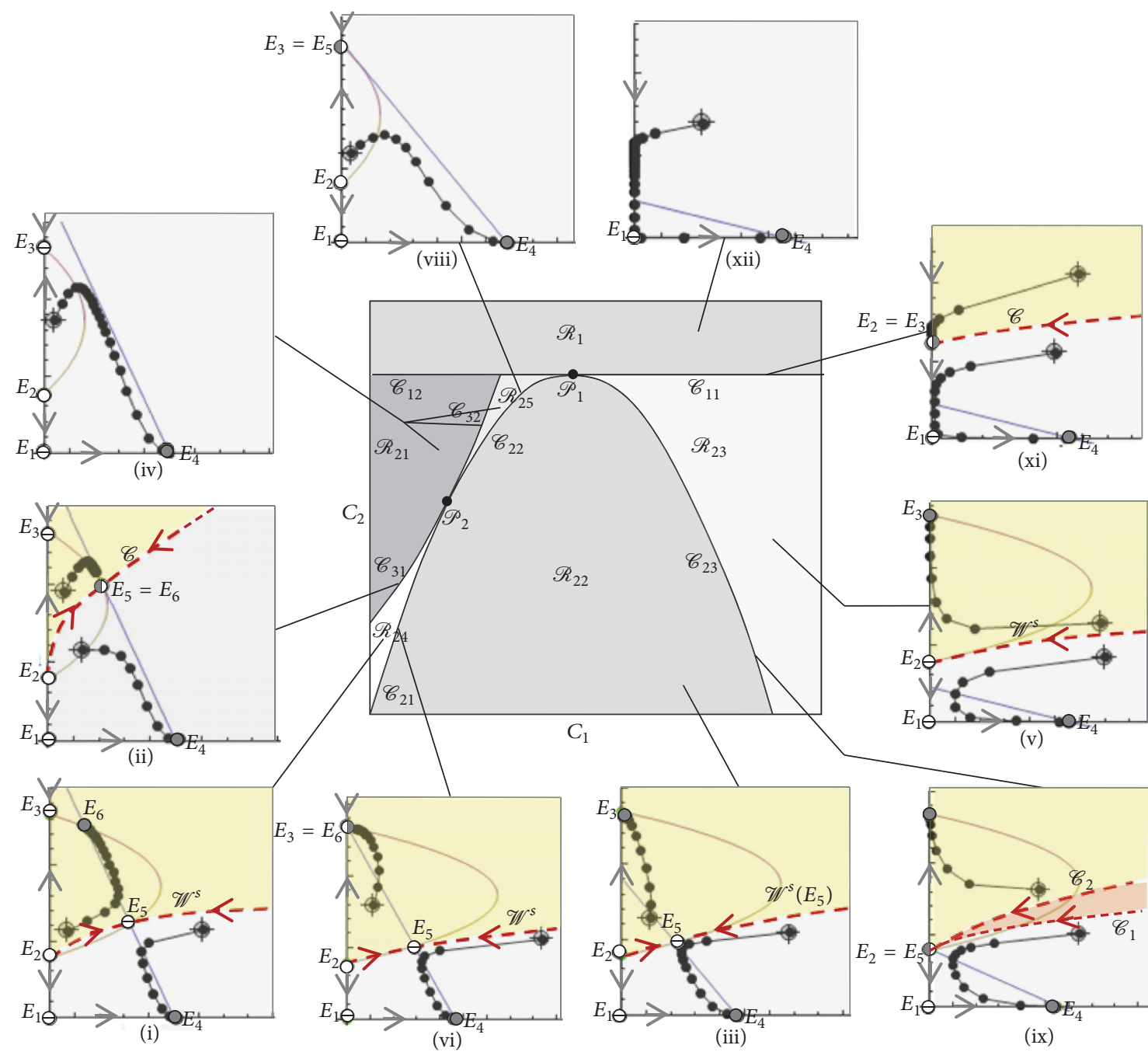

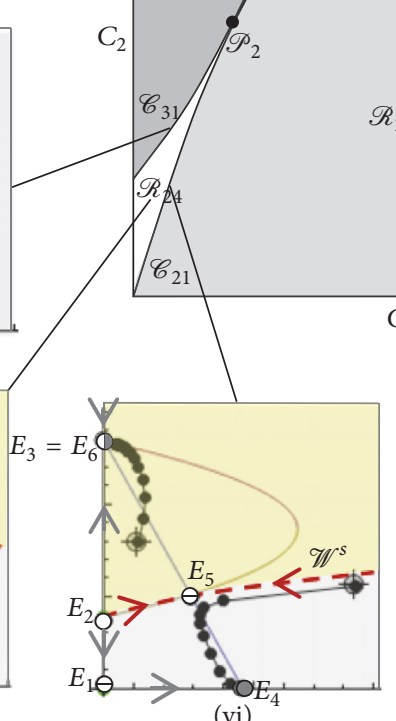

(vi)

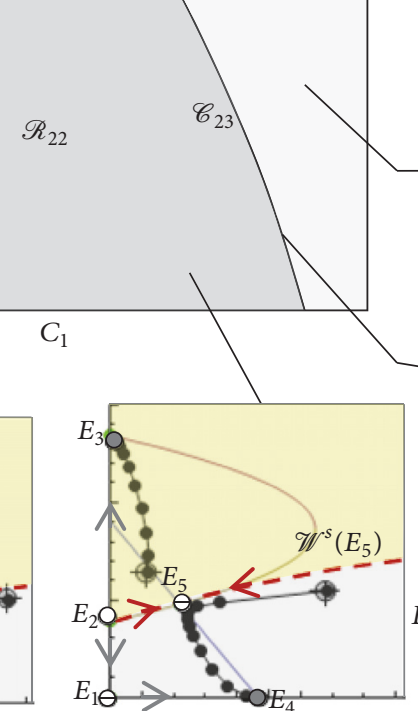

(iii)
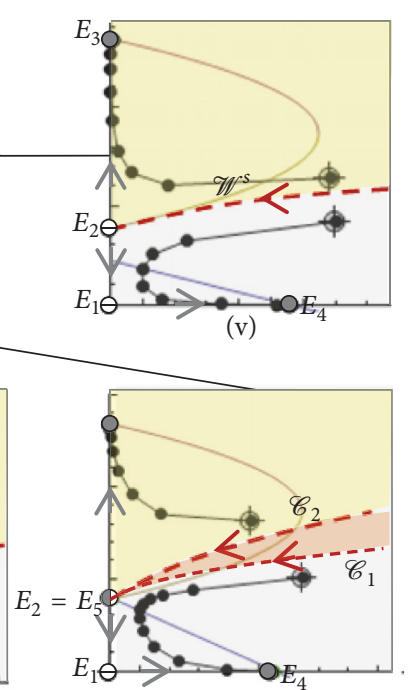

(ix)

(i) $A_{1}<1$

Figure 3: Parameter regions in terms of parameters $C_{1}$ and $C_{2}$ and corresponding dynamic scenarios for system (1) if $A_{1}<1$.

$\left.y<\left(1-\sqrt{1-4 A_{2} C_{2}}\right) /\left(2 A_{2}\right)\right\}$ and $\mathscr{W}^{s}\left(E_{3}\right)=$ $\left\{(0, y): y>\left(1-\sqrt{1-4 A_{2} C_{2}}\right) /\left(2 A_{2}\right)\right\}$. Take $\left(x_{0}, y_{0}\right) \in \mathscr{W}^{-} \cap \operatorname{int}\left(Q_{1}(0,0)\right)$. By Theorem 8 and Lemma 21 we have that there exists $n_{0}>0$ such that $T^{n}\left(x_{0}, y_{0}\right) \in \operatorname{int}\left(Q_{4}\left(E_{3}\right) \cap Q_{2}\left(E_{5}\right)\right), n>n_{0}$. In view of Corollary $4\left[\left[E_{1}, E_{6}\right]\right] \subseteq \mathscr{B}\left(E_{6}\right)$ and $\left[\left[E_{6}, E_{5}\right]\right] \subseteq \mathscr{B}\left(E_{6}\right)$. Since $T$ is competitive, this implies $\operatorname{int}\left(Q_{4}\left(E_{3}\right) \cap Q_{2}\left(E_{5}\right)\right)=\left[\left[E_{1}, E_{5}\right]\right] \subseteq \mathscr{B}\left(E_{6}\right)$. Take $\left(x_{0}, y_{0}\right) \in \mathscr{W}^{+} \cap \operatorname{int}\left(Q_{1}(0,0)\right)$. By Theorem 8 and Lemma 21 we have that there exists $n_{0}^{\prime}>0$ such that $T^{n}\left(x_{0}, y_{0}\right) \in \operatorname{int}\left(Q_{4}\left(E_{5}\right) \cap Q_{2}\left(E_{4}\right)\right), n>n_{0}^{\prime}$. Since $T$ is competitive, in view of Corollary $4 \operatorname{int}\left(Q_{4}\left(E_{5}\right) \cap\right.$ $\left.Q_{2}\left(E_{4}\right)\right)=\left[\left[E_{5}, E_{4}\right]\right] \subseteq \mathscr{B}\left(E_{4}\right)$. This completes the proof of statement (i).

(ii) Suppose $\left(C_{1}, C_{2}\right) \in \mathscr{C}_{31}$. By Proposition 11, in $Q_{1}(0,0)$ there exist five equilibria $E_{1}, E_{2}, E_{3}, E_{4}$, and $E_{5}=E_{6}$. By Theorem $19 E_{1}$ and $E_{3}$ are the saddle points, $E_{2}$ is repeller, and $E_{5}=E_{6}$ is nonhyperbolic. Similarly as in the proof of the statement (i), all conditions of Theorems 7, 8, and 9 are satisfied, which yields the existence of the invariant curve $\mathscr{C}$ with one endpoint at $E_{2}$ and which is passing through $E_{5}=E_{6}$, and it is graph of an increasing function. Let $\mathscr{W}^{-}=\{(x, y) \mid$ $(x, y) \preceq_{\text {se }}\left(\tilde{x}_{0}, \tilde{y}_{0}\right)$ for some $\left.\left(\tilde{x}_{0}, \tilde{y}_{0}\right) \in \mathscr{C}\right\}$ and $\mathscr{W}^{+}=$ $\left\{(x, y) \mid\left(\tilde{x}_{1}, \tilde{y}_{1}\right) \preceq_{\mathrm{se}}(x, y)\right.$ for some $\left.\left(\tilde{x}_{1}, \tilde{y}_{1}\right) \in \mathscr{C}\right\}$. By Lemma 21 and uniqueness of the global stable manifold we have $\mathscr{W}^{s}\left(E_{1}\right)=\{(0, y): 0 \leq y<$ $\left.\left(1-\sqrt{1-4 A_{2} C_{2}}\right) /\left(2 A_{2}\right)\right\}$ and $\mathscr{W}^{s}\left(E_{3}\right)=\{(0, y)$ : $\left.y>\left(1-\sqrt{1-4 A_{2} C_{2}}\right) /\left(2 A_{2}\right)\right\}$. Take $\left(x_{0}, y_{0}\right) \in \mathscr{W}^{-} \cap$ $\operatorname{int}\left(Q_{1}(0,0)\right)$. By Theorem 8 we have that there exists $n_{0}>0$ such that $T^{n}\left(x_{0}, y_{0}\right) \in \operatorname{int}\left(Q_{4}\left(E_{3}\right) \cap Q_{2}\left(E_{5}=\right.\right.$ $\left.\left.E_{6}\right)\right), n>n_{0}$. In view of Corollary $4 \operatorname{int}\left(Q_{4}\left(E_{3}\right) \cap\right.$ $\left.Q_{2}\left(E_{5}=E_{6}\right)\right)=\left[\left[E_{1}, E_{5}=E_{6}\right]\right] \subseteq \mathscr{B}\left(E_{5}=E_{6}\right)$. Take $\left(x_{0}, y_{0}\right) \in \mathscr{W}^{+} \cap \operatorname{int}\left(Q_{1}(0,0)\right)$. By Theorem 8 and Lemma 21 we have that there exists $n_{0}^{\prime}>0$ such that $T^{n}\left(x_{0}, y_{0}\right) \in \operatorname{int}\left(Q_{4}\left(E_{5}\right) \cap Q_{2}\left(E_{4}\right)\right), n>n_{0}^{\prime}$. Since 
$T$ is competitive, in view of Corollary $4 \operatorname{int}\left(Q_{4}\left(E_{5}\right) \cap\right.$ $\left.Q_{2}\left(E_{4}\right)\right)=\left[\left[E_{5}, E_{4}\right]\right] \subseteq \mathscr{B}\left(E_{4}\right)$. This completes the proof of statement (ii)

(iii) The proof is similar to the proof of case (i) and we skip it.

(iv) Suppose $\left(C_{1}, C_{2}\right) \in \mathscr{R}_{21} \cup \mathscr{R}_{25} \cup \mathscr{C}_{32}$. By Proposition 11 , in $Q_{1}(0,0)$ there exist four equilibria $E_{1}$, $E_{2}, E_{3}$, and $E_{4}$. By Theorem $19 E_{1}$ and $E_{3}$ are saddle points; $E_{2}$ is repeller; $E_{4}$ is locally asymptotically stable. By Lemma 21 and uniqueness of the global stable manifold we have $\mathscr{B}\left(E_{1}\right)=\mathscr{W}^{s}\left(E_{1}\right)=\{(0, y)$ : $\left.0 \leq y<\left(1-\sqrt{1-4 A_{2} C_{2}}\right) /\left(2 A_{2}\right)\right\}$ and $\mathscr{B}\left(E_{3}\right)=$ $\mathscr{W}^{s}\left(E_{3}\right)=\left\{(0, y): y>\left(1-\sqrt{1-4 A_{2} C_{2}}\right) /\left(2 A_{2}\right)\right\}$. Since, by Theorem 23 , every solution of system (1) converges to an equilibrium point, we have that $\mathscr{B}\left(E_{4}\right)=(0, \infty) \times[0, \infty)$.

(v) Suppose $\left(C_{1}, C_{2}\right) \in \mathscr{R}_{23}$. By Proposition 11, in $Q_{1}(0,0)$ there exist four equilibrium points $E_{1}, E_{2}, E_{3}$, and $E_{4}$. By Theorem $19 E_{1}$ and $E_{2}$ are the saddle points; $E_{3}$ and $E_{4}$ are locally asymptotically stable. By Lemma 21 and uniqueness of the global stable manifold we have $\mathscr{B}\left(E_{1}\right)=\mathscr{W}^{s}\left(E_{1}\right)=\{(0, y): 0 \leq y<$ $\left.\left(1-\sqrt{1-4 A_{2} C_{2}}\right) /\left(2 A_{2}\right)\right\}$, and $\{(0, y): y>(1-$ $\left.\left.\sqrt{1-4 A_{2} C_{2}}\right) /\left(2 A_{2}\right)\right\} \subseteq \mathscr{B}\left(E_{3}\right)$ and $\{(x, 0): x>0\} \subseteq$ $\mathscr{B}\left(E_{4}\right)$. Similarly as in the proof of case (i), all conditions of Theorems 7,8, and 9 are satisfied, which yields the existence of the global stable manifold $\mathscr{W}^{s}\left(E_{2}\right)$, which is a graph of an increasing function. The rest of the proof follows from the facts that $\mathscr{W}^{-}=\{(x, y) \mid$ $(x, y) \preceq_{\text {se }}\left(\tilde{x}_{0}, \tilde{y}_{0}\right)$ for some $\left.\left(\tilde{x}_{0}, \tilde{y}_{0}\right) \in \mathscr{W}^{s}\left(E_{2}\right)\right\}$ and $\mathscr{W}^{+}=\left\{(x, y) \mid\left(\tilde{x}_{1}, \tilde{y}_{1}\right) \preceq_{\text {se }}(x, y)\right.$ for some $\left(\tilde{x}_{1}, \tilde{y}_{1}\right) \in$ $\left.\mathscr{W}^{s}\left(E_{2}\right)\right\}$ are invariant sets, $E_{4} \in \mathscr{W}^{-}, E_{3} \in \mathscr{W}^{+}$, uniqueness of the global stable manifold $\mathscr{W}^{s}\left(E_{2}\right)$ and Theorem 23.

(vi) The proof is similar to the proof of case (i) and we skip it.

(vii) The proof is the same as the proof of case (viii) and we skip it.

(viii) Suppose $\left(C_{1}, C_{2}\right) \in \mathscr{C}_{22}$. By Proposition 11, in $Q_{1}(0,0)$ there exist four equilibrium points $E_{1}, E_{3}=$ $E_{5}, E_{2}$, and $E_{4}$. By Theorem 19 we have that $E_{4}$ is locally asymptotically stable; $E_{1}$ is a saddle point; $E_{3}=E_{5}$ is nonhyperbolic; and $E_{2}$ is repeller. By Lemma 21 and uniqueness of the global stable manifold we have $\mathscr{B}\left(E_{1}\right)=\mathscr{W}^{s}\left(E_{1}\right)=\{(0, y)$ : $\left.0 \leq y<\left(1-\sqrt{1-4 A_{2} C_{2}}\right) /\left(2 A_{2}\right)\right\}$. In view of Lemma 18, for $E_{3}=E_{5}$ we have that the eigenspace $E_{2}^{\lambda}$ associated with $\lambda_{2}$ is a coordinate axis, so we can not use Theorem 6. By Lemma 21 we obtain $\left\{(0, y): y>\left(1-\sqrt{1-4 A_{2} C_{2}}\right) /\left(2 A_{2}\right)\right\} \subseteq \mathscr{B}\left(E_{3}=\right.$ $E_{5}$ ). Similarly as in the proof of Theorem 8 (see [1] for more details) one can prove that for every $x \in \operatorname{int}\left(Q_{1}\left(E_{3}\right)\right)$ there exists $n_{0} \in \mathbb{N}$ such that $T^{n}(x) \in \operatorname{int}\left(Q_{4}\left(E_{3}\right)\right)$ for $n \geq n_{0}$. By Lemmas 21 and 22 for $\left(x_{0}, y_{0}\right) \in \operatorname{int}\left(Q_{4}\left(E_{3}\right)\right)$, there exists $t_{0}^{\prime}$ and $t_{0}^{\prime \prime}$ such that $E_{3} \preceq_{\mathrm{se}} M\left(t_{0}^{\prime}\right) \preceq_{\mathrm{se}}\left(x_{0}, y_{0}\right) \preceq_{\mathrm{se}}\left(t_{0}^{\prime \prime}, 0\right)$. Since
$E_{3} \preceq_{\mathrm{se}} T^{n}\left(M\left(t_{0}^{\prime}\right)\right) \preceq_{\mathrm{se}} T^{n+1}\left(M\left(t_{0}^{\prime}\right)\right) \preceq E_{4}$ we have that $T^{n}\left(M\left(t_{0}^{\prime}\right)\right) \rightarrow E_{4}$. Since $T^{n}\left(t_{0}^{\prime \prime}, 0\right) \rightarrow E_{4}$ we obtain $T^{n}\left(x_{0}, y_{0}\right) \rightarrow E_{4}$. This implies $\operatorname{int}\left(Q_{4}\left(E_{3}\right)\right) \subseteq \mathscr{B}\left(E_{4}\right)$, which completes the proof.

(ix) Suppose $\left(C_{1}, C_{2}\right) \in \mathscr{C}_{23}$. By Proposition 11, there exist four equilibrium points $E_{1}, E_{2}=E_{5}, E_{3}$, and $E_{4}$. By Theorem 19 we have that $E_{3}$ and $E_{4}$ are locally asymptotically stable; $E_{1}$ is a saddle point; $E_{2}=E_{5}$ is nonhyperbolic. By Lemma 21 and uniqueness of the global stable manifold we have $\mathscr{B}\left(E_{1}\right)=\mathscr{W}^{s}\left(E_{1}\right)=$ $\left\{(0, y): 0 \leq y<\left(1-\sqrt{1-4 A_{2} C_{2}}\right) /\left(2 A_{2}\right)\right\}$. In view of Lemma 18, for $E_{3}$, we have that $\lambda_{1}=1$ and $\lambda_{2}>1$, so we can not use Theorem 6 . By Lemmas 21 and 22 for $\left(x_{0}, y_{0}\right) \in \operatorname{int}\left(Q_{4}\left(E_{3}\right)\right)$, there exists $t_{0}^{\prime}$ and $t_{0}^{\prime \prime}$ such that $E_{3} \preceq_{\mathrm{se}} M\left(t_{0}^{\prime}\right) \preceq_{\mathrm{se}}\left(x_{0}, y_{0}\right) \preceq_{\mathrm{se}}\left(t_{0}^{\prime \prime}, 0\right)$. Since $E_{3} \preceq_{\mathrm{se}} T^{n}\left(M\left(t_{0}^{\prime}\right)\right) \preceq_{\mathrm{se}} T^{n+1}\left(M\left(t_{0}^{\prime}\right)\right) \preceq E_{4}$ we have that $T^{n}\left(M\left(t_{0}^{\prime}\right)\right) \rightarrow E_{4}$. Since $T^{n}\left(t_{0}^{\prime \prime}, 0\right) \rightarrow E_{4}$ we obtain $T^{n}\left(x_{0}, y_{0}\right) \rightarrow E_{4}$. This implies $\operatorname{int}\left(Q_{4}\left(E_{3}\right)\right) \subseteq \mathscr{B}\left(E_{4}\right)$. Let $\mathscr{C}_{1}$ denote the boundary of $\mathscr{B}\left(E_{4}\right)$ considered as a subset of $\operatorname{int}\left(Q_{1}\left(E_{2}\right)\right)$ and let $\mathscr{C}_{2}$ denote the boundary of $\mathscr{B}\left(E_{3}\right)$ considered as a subset of $\operatorname{int}\left(Q_{1}\left(E_{2}\right)\right)$. It is easy to see by using Lemmas 21 and 22 that $E_{2} \in \mathscr{C}_{1} \cap \mathscr{C}_{2}$. Since $T(\operatorname{int}(\mathscr{R})) \subset \operatorname{int}(\mathscr{R})$, following the proof of Claims 1 and 2 [20], one can see that $T\left(\mathscr{C}_{i}\right) \subseteq \mathscr{C}_{i}$ and $T^{n}\left(x_{0}, y_{0}\right) \rightarrow E_{2} \in \mathscr{C}_{i}$ for $\left(x_{0}, y_{0}\right) \in \mathscr{C}_{i}$ for $i=1,2$. Further, $\mathscr{C}_{i}$ are graphs of the continuous strictly increasing functions. If $\left(x_{0}, y_{0}\right)$ is point above the curve $\mathscr{C}_{1}$ and below the curve $\mathscr{C}_{2}$ then there exists $\left(x_{0}^{\prime}, y_{0}^{\prime}\right) \in \mathscr{C}_{1}$ and $\left(x_{0}^{\prime \prime}, y_{0}^{\prime \prime}\right) \in \mathscr{C}_{2}$ such that $\left(x_{0}^{\prime \prime}, y_{0}^{\prime \prime}\right) \preceq_{\mathrm{se}}\left(x_{0}, y_{0}\right) \preceq_{\mathrm{se}}\left(x_{0}^{\prime}, y_{0}^{\prime}\right)$. Since $T^{n}\left(x_{0}^{\prime \prime}, y_{0}^{\prime \prime}\right) \preceq_{\mathrm{se}} T^{n}\left(x_{0}, y_{0}\right) \preceq_{\mathrm{se}} T^{n}\left(x_{0}^{\prime}, y_{0}^{\prime}\right) \quad$ and $T^{n}\left(x_{0}^{\prime \prime}, y_{0}^{\prime \prime}\right) \rightarrow E_{2}$ and $T^{n}\left(x_{0}^{\prime}, y_{0}^{\prime}\right) \rightarrow E_{2}$ as $n \rightarrow \infty$ we have $T^{n}\left(x_{0}, y_{0}\right) \rightarrow E_{2}$ as $n \rightarrow \infty$.

(x) Suppose $\left(C_{1}, C_{2}\right) \in \mathscr{C}_{23}$. By Proposition 11, in $Q_{1}(0,0)$ there exist three equilibrium points $E_{1}, E_{2}=E_{3}$, and $E_{4}$. By Theorem $19 E_{2}=E_{3}$ is nonhyperbolic, $E_{1}$ is a saddle point, and $E_{4}$ is locally asymptotically stable. By Lemma 21 and uniqueness of the global stable manifold we have $\mathscr{B}\left(E_{1}\right)=\mathscr{W}^{s}\left(E_{1}\right)=\{(0, y)$ : $\left.0 \leq y<\left(1-\sqrt{1-4 A_{2} C_{2}}\right) /\left(2 A_{2}\right)\right\}$. In view of Lemma 18 we have that $\lambda_{1}=1$ and $\lambda_{2}<1$ if $C_{1}>$ $\left(1-A_{1}\right) /\left(2 A_{2}\right)$ and $\lambda_{2}>1$ if $C_{1}<\left(1-A_{1}\right) /\left(2 A_{2}\right)$, so we can use Theorem 6 if $C_{1}>\left(1-A_{1}\right) /\left(2 A_{2}\right)$. In this case there exists strictly increasing curve $\mathscr{C}$ with endpoint at $E_{2}=E_{3}$. The rest of the proof follows from Theorems 7, 8, and 9 and Lemma 21. Now, we assume that $C_{1}<\left(1-A_{1}\right) /\left(2 A_{2}\right)$. By Lemmas 21 and 22 for $\left(x_{0}, y_{0}\right) \in \operatorname{int}\left(Q_{4}\left(E_{3}\right)\right)$, there exists $t_{0}^{\prime}$ and $t_{0}^{\prime \prime}$ such that $E_{3} \preceq_{\mathrm{se}} M\left(t_{0}^{\prime}\right) \preceq_{\mathrm{se}}\left(x_{0}, y_{0}\right) \preceq_{\mathrm{se}}\left(t_{0}^{\prime \prime}, 0\right)$. Since $E_{3} \preceq_{\mathrm{se}} T^{n}\left(M\left(t_{0}^{\prime}\right)\right) \preceq_{\mathrm{se}} T^{n+1}\left(M\left(t_{0}^{\prime}\right)\right) \preceq E_{4}$ we have that $T^{n}\left(M\left(t_{0}^{\prime}\right)\right) \rightarrow E_{4}$. Since $T^{n}\left(t_{0}^{\prime \prime}, 0\right) \rightarrow E_{4}$ we obtain $T^{n}\left(x_{0}, y_{0}\right) \rightarrow E_{4}$. This implies int $\left(Q_{4}\left(E_{3}\right)\right) \subseteq \mathscr{B}\left(E_{4}\right)$. Let $\mathscr{C}$ denote the boundary of $\mathscr{B}\left(E_{4}\right)$ considered as a subset of $Q_{1}\left(E_{3}\right)$. It is easy to see by using Lemmas 21 and 22 that $E_{3} \in \mathscr{C}$. Since $T(\operatorname{int}(\mathscr{R})) \subset \operatorname{int}(\mathscr{R})$, following the proof of Claims 1 and 2 [20], one can see that $T(\mathscr{C}) \subseteq \mathscr{C}$. Further, $\mathscr{C}$ is graph of strictly 
increasing function. By Theorem 23 if $\left(x_{0}, y_{0}\right) \in \mathscr{C}$ then $T^{n}\left(x_{0}, y_{0}\right) \rightarrow E_{3} \in \mathscr{C}$. If $\left(x_{0}, y_{0}\right)$ is point above the curve $\mathscr{C}$ then there exists $\left(x_{0}^{\prime}, y_{0}^{\prime}\right)$ and $y_{0}^{\prime \prime}>\bar{y}_{3}$ such that $\left(0, y_{0}^{\prime \prime}\right) \preceq_{\mathrm{se}}\left(x_{0}, y\right) \preceq_{\mathrm{se}}\left(x_{0}^{\prime}, y_{0}^{\prime}\right)$. Since $T^{n}\left(0, y_{0}^{\prime \prime}\right) \preceq_{\mathrm{se}} T^{n}\left(x_{0}, y_{0}\right) \preceq_{\mathrm{se}} T^{n}\left(x_{0}^{\prime}, y_{0}^{\prime}\right), T^{n}\left(0, y_{0}^{\prime \prime}\right) \rightarrow$ $E_{3}$, and $T^{n}\left(x_{0}^{\prime}, y_{0}^{\prime}\right) \rightarrow E_{3}$ as $n \rightarrow \infty$ we have $T^{n}\left(x_{0}, y_{0}\right) \rightarrow E_{3}$ as $n \rightarrow \infty$.

Based on a series of numerical simulations we propose the following conjectures.

Conjecture 26. Suppose that all assumptions of the statement (ix) of Theorem 25 are satisfied; then the following holds:

$$
\mathscr{B}\left(E_{2}\right)=\mathscr{C}_{1}=\mathscr{C}_{2} \text {. }
$$

Conjecture 27. Assume that $C_{1}, C_{2} \in \mathscr{P}_{1}$. There exist three equilibrium points $E_{1}, E_{3}=E_{2}=E_{5}$, and $E_{4}$, where $E_{4}$ is locally asymptotically stable; $E_{1}$ is a saddle point; and $E_{3}=$ $E_{2}=E_{5}$ is nonhyperbolic. The basins of attraction of $E_{1}$ and $E_{3}=E_{2}=E_{5}$ are given by $\mathscr{B}\left(E_{1}\right)=\{(0, y): 0 \leq$ $\left.y<\left(1-\sqrt{1-4 A_{2} C_{2}}\right) /\left(2 A_{2}\right)\right\}$ and $\mathscr{B}\left(E_{3}\right)=\{(0, y): y>$ $\left.\left(1-\sqrt{1-4 A_{2} C_{2}}\right) /\left(2 A_{2}\right)\right\}$ and the basin of attraction of $E_{4}$ is $\mathscr{B}\left(E_{4}\right)=\{(x, y): x>0, y \geq 0\}$.

\section{Conflicts of Interest}

The authors declare that there are no conflicts of interest regarding the publication of this paper.

\section{References}

[1] M. R. Kulenović and O. Merino, "Invariant manifolds for competitive discrete systems in the plane," International Journal of Bifurcation and Chaos in Applied Sciences and Engineering, vol. 20, no. 8, pp. 2471-2486, 2010.

[2] J. M. Cushing, S. Levarge, N. Chitnis, and S. M. Henson, "Some discrete competition models and the competitive exclusion principle," Journal of Difference Equations and Applications, vol. 10, no. 13-15, pp. 1139-1151, 2004.

[3] M. R. Kulenović and O. Merino, "Competitive-exclusion versus competitive-coexistence for systems in the plane," Discrete and Continuous Dynamical Systems. Series B. A Journal Bridging Mathematics and Sciences, vol. 6, no. 5, pp. 1141-1156, 2006.

[4] A. Brett and M. R. Kulenovic, "Two species competitive model with the Allee effect," Advances in Difference Equations, vol. 2014, article 307, 2014.

[5] A. Brett and M. R. Kulenović, "Basins of attraction for twospecies competitive model with quadratic terms and the singular Allee effect," Discrete Dynamics in Nature and Society, Article ID 847360, 16 pages, 2015.

[6] D. Burgić, M. R. Kulenović, and M. Nurkanović, "Global dynamics of a rational system of difference equations in the plane," Communications on Applied Nonlinear Analysis, vol. 15, no. 1, pp. 71-84, 2008.

[7] D. Clark and M. R. Kulenović, "A coupled system of rational difference equations," Computers \& Mathematics with Applications, vol. 43, no. 6-7, pp. 849-867, 2002.
[8] J. E. Franke and A.-A. Yakubu, "Mutual exclusion versus coexistence for discrete competitive systems," Journal of Mathematical Biology, vol. 30, no. 2, pp. 161-168, 1991.

[9] J. E. Franke and A.-A. Yakubu, "Geometry of exclusion principles in discrete systems," Journal of Mathematical Analysis and Applications, vol. 168, no. 2, pp. 385-400, 1992.

[10] M. W. Hirsch and H. Smith, "Chapter 4 Monotone Dynamical Systems," Handbook of Differential Equations: Ordinary Differential Equations, vol. 2, pp. 239-357, 2006.

[11] S. Kalabušić, M. R. Kulenović, and E. Pilav, "Multiple attractors for a competitive system of rational difference equations in the plane," Abstract and Applied Analysis, vol. 2011, Article ID 295308, 35 pages, 2011.

[12] R. M. May and W. J. Leonard, "Nonlinear aspects of competition between three species," SIAM Journal on Applied Mathematics, vol. 29, no. 2, pp. 243-253, 1975.

[13] H. L. Smith, "Invariant curves for mappings," SIAM Journal on Mathematical Analysis, vol. 17, no. 5, pp. 1053-1067, 1986.

[14] H. L. Smith, "Periodic competitive differential equations and the discrete dynamics of competitive maps," Journal of Differential Equations, vol. 64, no. 2, pp. 165-194, 1986.

[15] H. L. Smith, "Periodic solutions of periodic competitive and cooperative systems," SIAM Journal on Mathematical Analysis, vol. 17, no. 6, pp. 1289-1318, 1986.

[16] H. L. Smith, "Planar competitive and cooperative difference equations," Journal of Difference Equations and Applications, vol. 3, no. 5-6, pp. 335-357, 1998.

[17] P. de Mottoni and A. Schiaffino, "Competition systems with periodic coefficients: a geometric approach," Journal of Mathematical Biology, vol. 11, no. 3, pp. 319-335, 1981.

[18] P. Hess, Periodic-Parabolic Boundary Value Problems and Positivity, vol. 247 of Pitman Research Notes in Mathematics Series, Longman Scientific \& Technical, Harlow, UK, 1991.

[19] S. Basu and O. Merino, "On the global behavior of solutions to a planar system of difference equations," Communications on Applied Nonlinear Analysis, vol. 16, no. 1, pp. 89-101, 2009.

[20] V. Hadžiabdić, M. R. Kulenović, and E. Pilav, "Dynamics of a two-dimensional competitive system of rational difference equations with quadratic terms," Advances in Difference Equations, vol. 301, p. 32, 2014. 


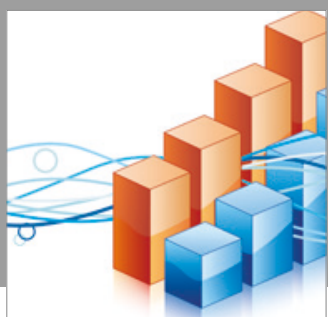

Advances in

Operations Research

vatersals

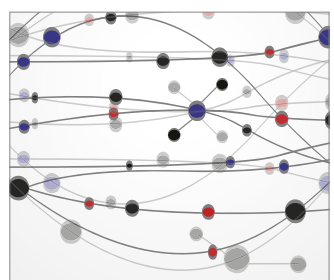

\section{The Scientific} World Journal
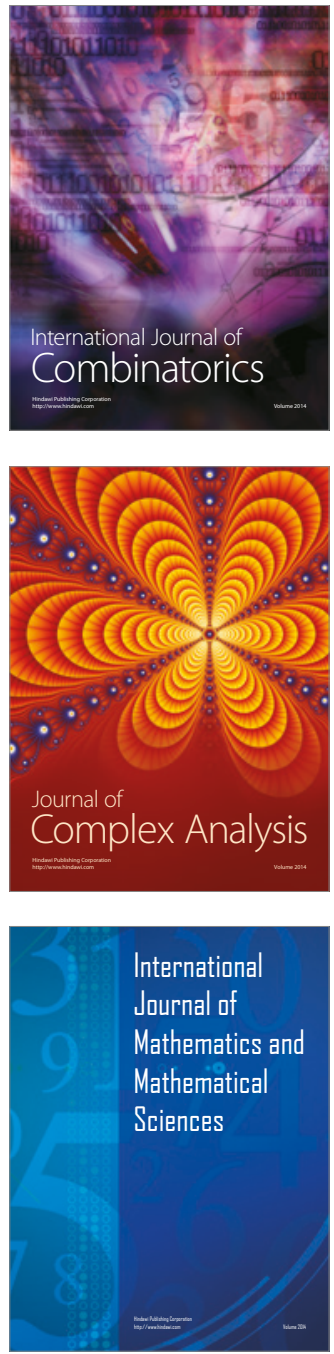
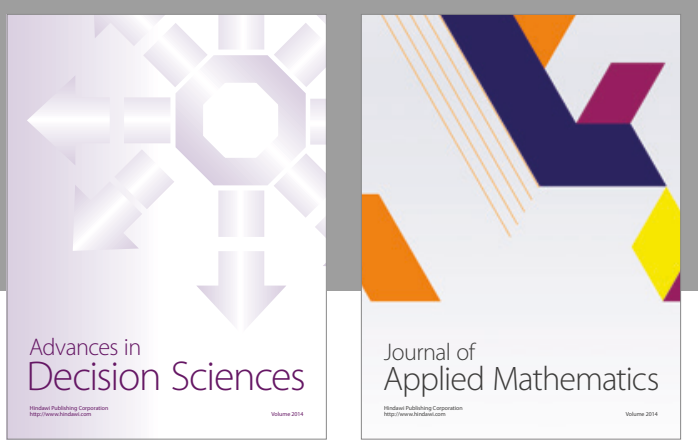

Algebra

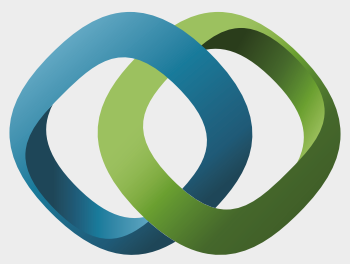

\section{Hindawi}

Submit your manuscripts at

https://www.hindawi.com
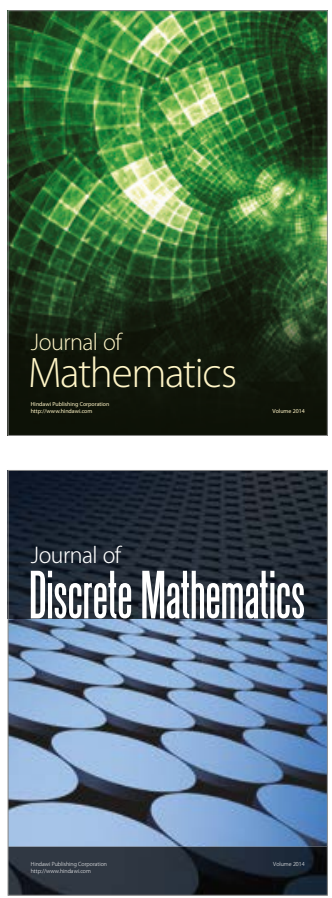

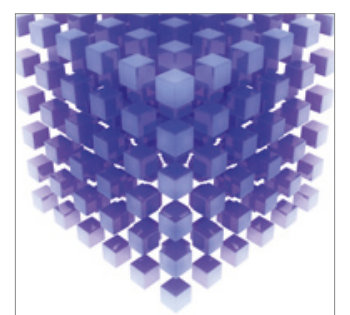

Mathematical Problems in Engineering
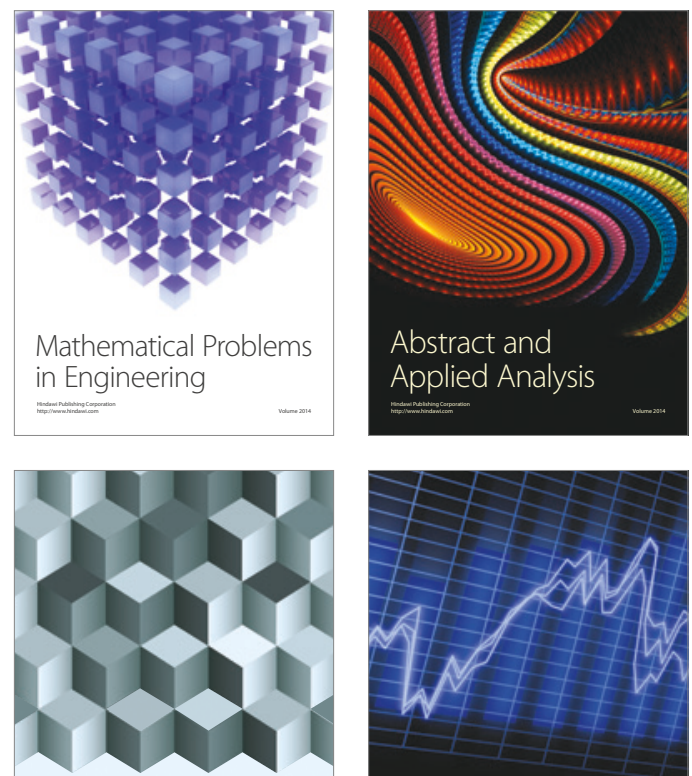

Journal of

Function Spaces

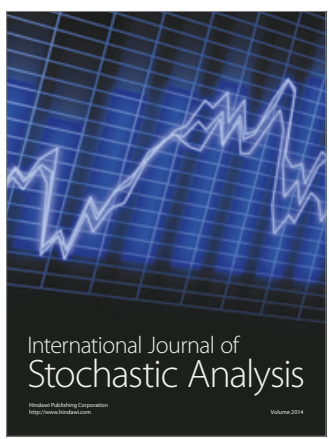

Probability and Statistics
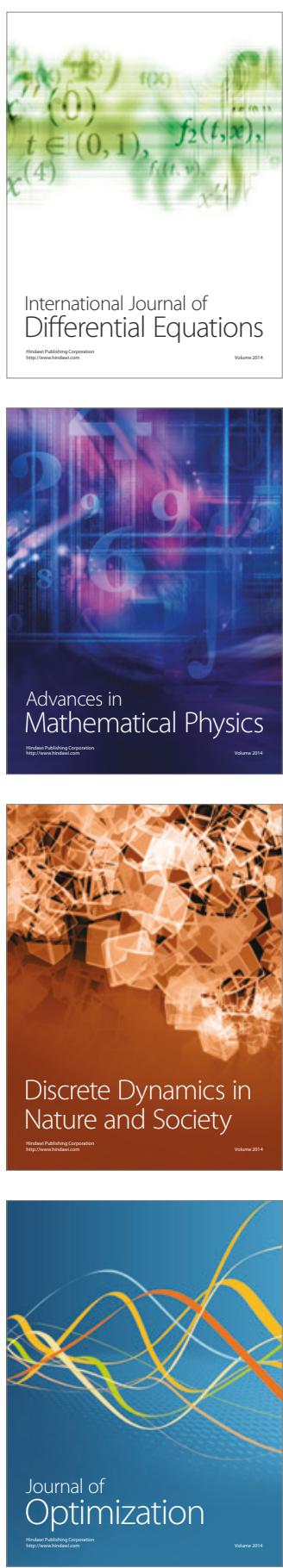